\title{
What came out of visual memory: Inferences from decay of difference-thresholds
}

\author{
Thomy Nilsson ${ }^{1}$ \\ Published online: 28 April 2020 \\ (C) The Psychonomic Society, Inc. 2020
}

\begin{abstract}
Means and standard deviations of psychophysical measurements are first and second statistical moments that reflect neural mechanisms underlying the measured sensation: for example, standard deviations of hue matches are difference-thresholds that reflect the color pathways' spectral response. Might memory psychophysics yield similar insight into neural mechanisms underlying recognition? Five experiments measured memory means and difference-thresholds: Means of monochromatic hue matches made from memory hardly changed with memory time. Hue memory remained consistent even though the variability of those matches increased exponentially as memory time increased to $14 \mathrm{~s}$. This indicates the loss of accuracy in the memory resulted from a random decay of the stored information. Nevertheless, the hue memory difference-thresholds as a function of wavelength continued to resemble sensory difference-threshold functions as memory time increased. Similar results obtained by dichoptic matches indicated this resemblance was not a retinal effect. Line and grating orientation memory difference-thresholds also retained their similarity to sensory difference-threshold functions as memory time increased. The similarity was not some artifact of measuring difference-thresholds by matching because such results were also obtained using a forced-choice method. Similarity of memory discrimination functions to sensory functions is quantitative evidence that, despite decay, activity retrieved from memory resembles the sensory responses used when two stimuli are compared simultaneously. Feasibility of this conclusion was tested by modeling the memory-matching procedure. It produced difference-thresholds resembling those obtained with simultaneous matches when the stored signal had decayed to $10 \%$. Together these results provide "inside" evidence that cortical activity resembling sensory responses underlie recognition.
\end{abstract}

Keywords Visual working memory $\cdot$ Difference-thresholds $\cdot$ Hue $\cdot$ Linear orientation $\cdot$ Neural model

\section{Introduction}

In the early days of experimental research on memory, recognition and recall were considered integral with perception and consciousness. "Since the days of Locke, we may say, the notion of memory as a faint likeness of perception, and of 'reproduction' as a revival through imagery, has been a common one in psychology." Bentley $(1899$, p. 2) continues, "Bearing in mind this fact, we have tried to determine the exact place of the image in the memory consciousness, and have then endeavored to discover the changes which the image undergoes in the course of time." Over a hundred years later, the ability to record and stimulate activity in the human

Thomy Nilsson

nilsson@upei.ca

1 Psychology Department, University of Prince Edward Island, 550 University Avenue, Charlottetown, PEI C1A 4P3, Canada brain enables saying that imagery and working memory "have similar effects on early visual excitability" (Cattaneo, Vecchi, Pascual-Leone, \& Silvanto, 2009, p. 1393), and that "Retention of features in visual short-term memory involves maintenance of sensory traces in early visual cortex" (Sneve et al., 2015, p. 1). The research reported here is based on the premise that measuring precisely the change in what is recalled can provide insight into what is retrieved and how recognition occurs. Such measurements are still needed.

Evidence is now accumulating that recall of visual stimuli is accompanied by sensory-specific neural activity in the visual and prefrontal cortex (e.g., Fuster, 1997; Killebrew et al., 2018; Postle, 2015; Schurgin, 2018; Serences, 2016). Furthermore, that cortical stimulation can alter accuracy of subsequent recall (e.g., Rademaker, van den Ven, Tong, \& Sack, 2017; Tonegawa, Liu, Ramirez, \& Redondo, 2015) demonstrates a causal link between changes in memory activity and what is recalled. Psychophysical experiments on various kinds of visual memories show that the accuracy of visual 
memories deteriorates over time (e.g., Cornelissen \& Greenlee, 2000; Lee \& Harris, 1996; Petrusic, Harrison, \& Baranski, 2004; Ricker, Spiegel, \& Cowan, 2014). Whatever the physiological basis of the memory activity that stems from a sensory response, the loss of recall accuracy can be construed as being due to several influences that, together, infuse growing randomness and possibly some change in that activity (e.g., Ricker et al., 2014; Schneegans \& Bays, 2018; Skottun, 2004).

Psychophysical threshold measurements have long provided insight into the sensory process mediating perception (Blackwell, 1972; Graham, 1989; Riggs \& Wooten, 1972). At times these insights have preceded direct physiological verification. For example, Blakemore and Campbell (1969) used the first-moment statistical means of adaptation thresholds to show evidence of spatial frequency tuning before Maffei and Fiorentini (1973) observed visual cortex neurons with these characteristics. Using a similar method, Nilsson, Richmond, and Nelson (1975) found evidence of temporal frequency tuning before this was verified by Foster, Gaska, Nagles, and Pollen's (1985) recordings from the visual cortex.

Yet a quantitative approach to memory as an experience is problematic with the most common types of information words and pictures (Laming \& Scheiwiller, 1985). There is no simple way to measure how different one word or one picture is from another. For example, recalling "cat" when asked to remember "rat" is wrong, but it is not as wrong as recalling "cow." The hue qualities of color are salient, complex, and memorable experiences that can be measured in various scales such as wavelength, the CIE-LAB system, the Munsell system, or the Natural Color System, depending on how the colors are produced and selected. Experiments have used various methods to measure the remembered colors of wavelengths (e.g., Collins, 1932), light mixtures (e.g., Bae, Olkkonen, Allred, \& Flombaum, 2015), and pigmented surfaces (e.g., Bloj, Weiss, \& Gegenfurtner, 2016). Prior to Brown and Wald's (1964) and Marks, Dobelle, and MacNichol's (1964) direct measurement of the spectral absorption of the visual color receptors, second-moment difference-thresholds by Stiles (1959) and Wright and Pitt (1934) were used to estimate these mechanisms of color vision. Their inferences from these psychophysical measurements proved accurate (Bouman, \& Walraven, 1972; Kaiser \& Boynton, 1996; Pokorny \& Smith, 2004). Likewise, differencethreshold measurements by Chapanis (1951) and Jastrow (1892) for line orientation revealed the oblique effect well before Hubel and Weisel (1959) mapped the distribution of linear receptive-field cells in the visual cortex. Nilsson (1979) showed how two-flash interval difference-thresholds can reveal the visual impulse response.

On that basis, Nilsson and Nelson (1981) sought insight into the neural activity underlying memory of a basic visual sensation by measuring how hue difference-thresholds fared over time in memory. To control memory time, their observers used an iterative procedure to match the hue of 1-s presentations of 16 monochromatic wavelengths from 425 to $650 \mathrm{~nm}$ while viewed simultaneously and after delays from 0.1 to $24 \mathrm{~s}$. They found that the average matched wavelengths differed by about a just-noticeable difference (jnd) from the remembered stimulus' wavelength, regardless of memory time. Sometimes longer and sometimes shorter than the wavelength of the remembered hue, the matches on average were almost a flat function as memory time increased. This is consistent with other visual memory experiments that find little or no shift in color memory over time (e.g., Allen, Beilock, \& Shevell, 2011; Nemes, Parry, \& McKeefry, 2010). However, the averages provided little insight except to suggest that memory for color was very accurate over time periods that could be interpreted as possibly extending beyond short-term memory.

A different picture emerged when Nilsson and Nelson (1981) looked at the variability of the matches in terms of standard deviations. These increased exponentially as a function of memory time. Assuming the increasing variability reflected a decay function, this suggested a half-life (a doubling of the variability) of about $24 \mathrm{~s}$. For the simultaneous matches, the standard deviations were sensory differencethresholds (Woodworth \& Schlosberg, 1954), which varied as a function of wavelength in the characteristic manner of hue difference-thresholds (Graham, 1965). Likewise, the standard deviations of the delayed matches could be considered memory difference-thresholds. Based on the assumption that the decay was due to increasing randomness in the memory traces, they expected the difference-threshold functions would become less distinct as memory time increased. That was not the case. Though the memory difference-thresholds altogether increased with time, they maintained the distinct shape of the sensory difference-threshold function.

When subjects match one hue to another, they adjust the wavelength of one until it produces a sensory response that is similar to the other's. Since matching viewed hues with remembered hues produced a difference-threshold function similar to that obtained by matching two simultaneously viewed hues, Nilsson and Nelson (1981) concluded that the activity that came out of memory was similar to the sensory response produced by those hues. This conclusion is now consistent with the above more recent evidence that recall of a stimulus is accompanied by cortical activity in similar locations or by similar patterns of activity as when those stimuli are viewed. Similar responses during sensory and memory processes are also consistent with models of recognition based on correlational operations (e.g., Austin \& Lees, 2000; Dara, Satyanarayana, \& Govardhan, 2014; Metcalfe, 1991; Mewhort \& Johns, 2005; Pribram, Nuwer, $\&$ Baron, 1974). Similar sensory and memory processes are also consistent with evidence that consciousness and imagery are mediated by cortico-thalamic and cortico-cortical 
loops (e.g., Guo et al., 2017; Pergola, Ranft, Mathias, \& Suchan, 2013).

While several other studies have measured memory for color, most have used a single memory time of a few seconds or less (e.g., Bae, Olkkonen, Allred, \& Flombaum, 2015; Bloj, Weiss, \& Gegenfurtner, 2016). This seems short-sighted given other evidence that visual memories persist at least for $8 \mathrm{~s}$ (Parks, Kroll, Salzberg, \& Parkinson, 1972). Furthermore, measurements at a single memory time cannot show how memory changes over time. A few studies have used longer memory times (e.g., Collins, 1932; Perez-Carpinell, Baldovi, de Fez, \& Castro, 1998), but others (e.g., Rademaker, Park, Sack, \& Tong, 2018) do not present data for enough colors to infer difference-threshold functions.

As discussed above, sensory difference-thresholds directly reflect the neural activity that underlies the sensory responses making those discriminations possible. Similar reasoning applied to memory difference-thresholds implies that they reflect the neural activity that occurs when one recognizes the similarity of a memory to what was seen. The resemblance of memory difference-thresholds to sensory differencethresholds found by Nilsson and Nelson (1981) indicates that the activity in memory is similar to the sensory activity that produced the memory. This paper reports a series of experiments to answer several questions arising from that study:

1. The similarity of memory difference-threshold functions to visual difference-threshold functions was based on results averaged across four observers. Similar results found by Seliger (2002) after a 5-s delay were also based on subject averages. Could the similarity of sensory to memory difference-thresholds be an artifact of data averaging, or would more data from individual observers also show this effect?

2. The matches were made monocularly with both the stimulus being remembered and the stimulus being adjusted presented to the same eye. Therefore the results could be due to some persisting retinal adaptation or assimilation effect rather than memory. What would an experiment using dichoptic presentation of the two stimuli show?

3. As a qualitative effect evoked by various wavelengths of light, hue is less directly related to image geometry, which is linked to anatomical mapping. Would memory difference-thresholds for a spatial characteristic such as line orientation remain similar to the sensory differencethresholds?

4. The above experiments measured difference-thresholds in terms of the variability of stimulus matches. Was the memory effect somehow an artifact of the matching procedure?

5. The line-orientation memory functions were more variable than the hue memory functions. Being narrower than the image of the monochromator slits, the image of a fine line on the retina would be more susceptible to local variations in astigmatism, and location of focus. Spatial gratings have a more extended image, and spatial frequency tuning is a primary basis of shape and scene perception (Blakemore \& Campbell, 1969; De Valois, De Valois, \& Yund, 1979). Would the sensory memory effect be more evident if orientation difference-thresholds were measured using spatial gratings?

6. If memory is based on a stored sensory response, how can the difference-threshold functions retain their shape as that stored response becomes more random over time? To find out if this was even possible, an operating model of the memory matching procedure was constructed and tested with various rates of decay and processing noise.

\section{General method}

The following experiments employed undergraduate students aged 17-24 years, who gave their informed consent to participate as paid observers and/or research assistants. The procedures were in accordance of the Code of Ethics of the World Medical Association (Declaration of Helsinki) and approved by the University of Prince Edward Island's Ethics Committee for Human Research. Observers were screened for normal color vision using the Dvorine test and for normal or corrected acuity and astigmatism using a Snellen chart. Depending on their previous experience, each observer had $3-5 \mathrm{~h}$ of practice. The observers were seated in a dim, sound-deadened booth separated from the experimenter and apparatus. All matches were made while using a bite-board system to maintain head position. The hue memory experiments used Maxwellian viewing of monochromator exit slits with 4-mm artificial pupils. They were operated by manual remote controls and electronic timers. The line and grating orientation experiments used binocular viewing of mechanical lines displayed in a tachistoscope or gratings displayed on a monitor. They were computer automated.

The iterative stimulus matching procedure was similar to that used by Nilsson and Nelson (1981): All stimuli had a duration of $1 \mathrm{~s}$. For sensory difference-thresholds, monochromatic stimuli were presented simultaneously while linear orientation stimuli were presented successively. Both were repeated after $1 \mathrm{~s}$. For memory difference-thresholds, the stimuli were separated by delays from 0.1 up to $24 \mathrm{~s}$ and the sequence repeated after $1.1 \mathrm{~s}$ or after a delay equal to the memory time. With practice, the observers used about 12 iterations to obtain hue matches - slightly fewer for orientation matches. The various stimulus conditions and memory times were tested in counterbalanced pseudo-random orders so that both the stimulus and memory time changed for successive measurements. Except for Experiment 4, the difference-thresholds 
were defined as the standard deviations of the hue and orientation matches. Normalized averaging was used to combine data from several observers (Etzkorn, 2012). Assuming that the data are samples of continuous functions, first-order digital filtering was applied to make trends clearer across wavelengths or orientation angles (Cook \& Miller, 1992).

\section{Experiment 1: Monochromatic hue memory}

This study replicated Nilsson and Nelson's (1981) experiment with more wavelengths, matches, and observers, though only for memory times up to $14 \mathrm{~s}$. With more measurements per observer, it sought to determine if the similarity of memory difference-threshold functions to sensory functions would be evident in the data of individual observers.

\section{Method}

Six observers made eight matches of 20 (435 (violet), 455, 465 ..., 635 (red $) \mathrm{nm}$ ) wavelengths viewed simultaneously and after delays of 2, 3, 5, 8, and $14 \mathrm{~s}$. The optical system was based on a Bausch \& Lomb "Hi Intensity" grating monochromator with a 10-nm half-intensity band width and an observercontrolled device that enabled shifting the lower half of the output spectrum by $\pm 30 \mathrm{~nm}$ (Nilsson, 1971). The stimuli were equated in brightness by heterochromatic matching to the $575 \mathrm{~nm}$ (yellow) stimulus set at $3 \mathrm{log}$ above photopic threshold. As the observer adjusted wavelength to obtain a hue match, an analog computer maintained constant brightness by adjusting a neutral density wedge. The stimuli appeared in a $1.5 \times 0.5^{\circ}$ visual-angle vertical slit divided by a $0.5^{\circ}$ black gap that contained a dim white central fixation light (see Fig. 1). This configuration was used to minimize contrast and assimilation interactions between the two stimuli.

\section{Results}

To see whether storing a hue in memory changed its hue, the wavelength of the hue to be matched was subtracted from the

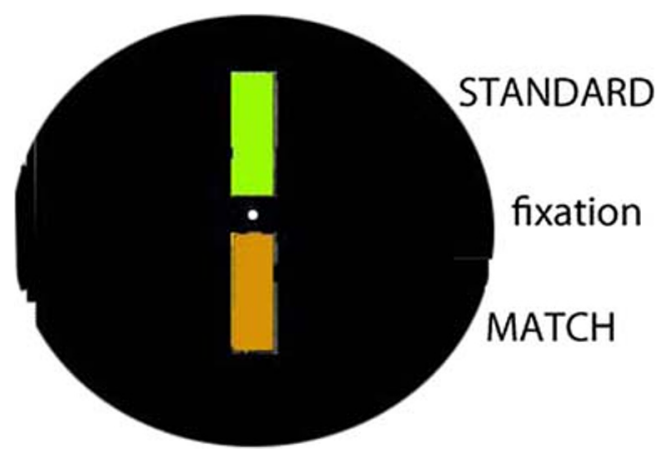

Fig. 1 Visual field for the hue matching measurements mean wavelength of eight matches that were made simultaneously and after delays of 2-14 s. Figure 2 shows the results from observer CAS for each of the 20 wavelengths. Shifts in hue of the simultaneous matches were generally smaller than shifts of the delayed matches. For the simultaneous matches, the average magnitude of the differences across wavelength equaled $0.21 \mathrm{~nm}$ versus $0.30-0.38 \mathrm{~nm}$ for the delayed matches as noted by the numbers on the left of each function. Analyzed as a function of wavelength, these shifts were not consistent across memory time. This was determined by using correlations to measure the similarity of the delayed functions to the simultaneous functions. These correlations equaled +0.09 , $+0.21,+0.04,+0.13$, and +0.09 at the respective delays of 2 , $3,5,8$, and $14 \mathrm{~s}$ (at 18 d.f., $95 \%$ confidence level for significant similarity $=+0.44$ ). All 120 of the CAS mean matches had a mean correlation of +0.14 with those of the other five observers (at 118 d.f. ( 20 wavelengths $* 6$ delays, minus two), $95 \%$ confidence $=+0.18$ ). In other words, there was no consistent evidence that the hues shifted in memory.

Figure 3 shows CAS' and the average of all six observers' hue difference-thresholds as a function of wavelength when the stimuli were viewed simultaneously and after delays of from 2-14 s. As time in memory increased, the differencethreshold functions did not become more random. Similarity of the memory functions to the bottom simultaneously matched sensory function was still evident after $14 \mathrm{~s}$. This apparent similarity is supported by +0.59 and +0.80 respective average correlations of CAS' and all six observers' memory functions to their sensory function (comparing 20 wavelength functions, $99 \%$ confidence of similarity at 18 d.f. $=+0.56$ ).

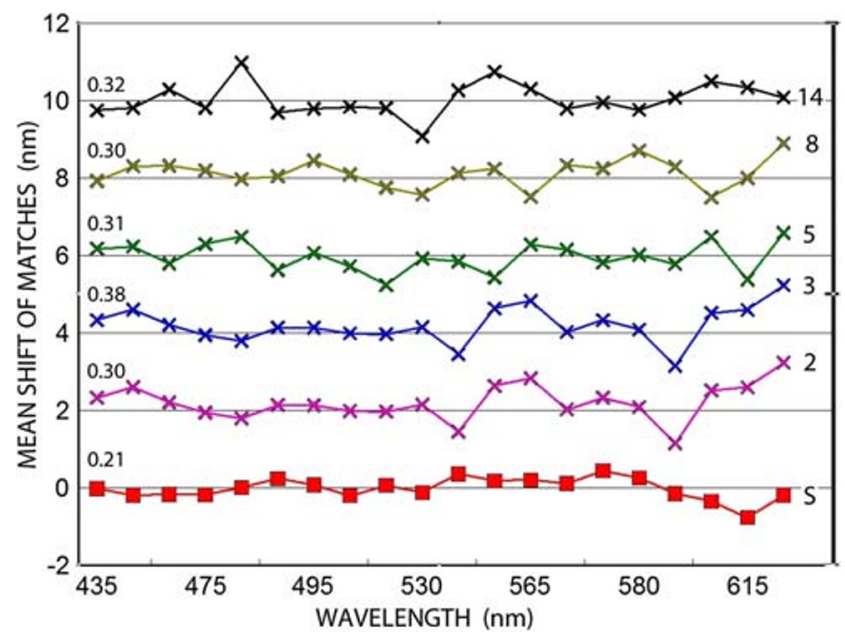

Fig. 2 Hue shifts of the matches. Mean wavelength difference of eight hue matches for observer CAS from the wavelength of the hue being matched, as a function of wavelength when viewed simultaneously (S) and after delays of 2, 3, 5, 8, and $14 \mathrm{~s}$ as labeled along the right side of the graph. The delayed functions are successively offset by $2 \mathrm{~nm}$ to prevent overlap. The numbers on the left end of each function are the average magnitudes of the difference in wavelength between the hue to be matched and the observer's matches, further averaged across the wavelengths at that delay 


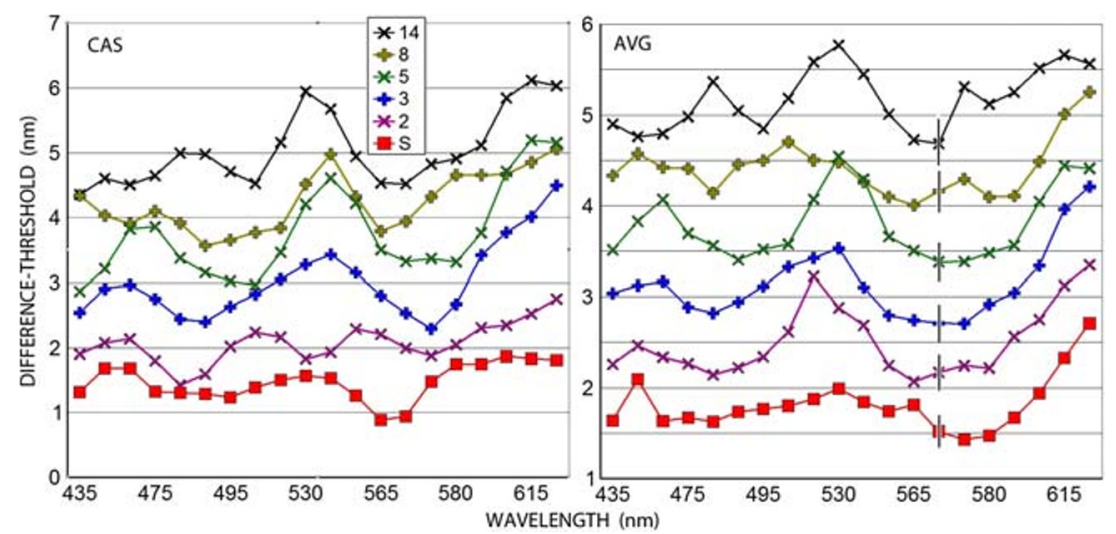

Fig. 3 How accuracy of memory for 20 hues changed over time. Hue difference-thresholds for observer CAS and the average of all six observers are shown as a function of wavelength when hues were matched simultaneously $(\mathrm{S})$ and after delays of 2-14 s. The functions for each

Because difference-thresholds are based on variability of the matches, it is only possible to calculate the variability of the average observer difference-thresholds. Standard error of the average observer functions is based on the standard error of the six difference-thresholds - one from each observer at each wavelength by delay time condition. These standard errors equalled about $10 \%$ or less of the average differencethreshold at any wavelength $\times$ delay-time condition (overall mean standard error $=0.21 \mathrm{~nm}$, overall mean differencethreshold $=2.3 \mathrm{~nm}$ ). For simplicity, vertical standard error bars at the $575-\mathrm{nm}$ position are plus the average of the standard errors across wavelengths for that memory time. Standard error of the difference-thresholds varied little as a function of memory time.

To see the general trend of how accuracy of the hue matches changed over time, the difference-thresholds were averaged across wavelength for the simultaneous and the delayed matches. These results are shown in Fig. 4 for each of the six observers and their average. The graphs are consistent with an exponential decay function that has a half-life greater than $14 \mathrm{~s}$. The apparent similarity of these decay functions is borne out by the +0.72 average correlation of the other five observers' decay functions with CAS' decay function (at 4 d.f., $95 \%$ confidence of similarity $=+0.71$ ).

\section{Discussion}

The absence of systematic shifts in hue matches over time indicates that memories of hue are not subject to a predisposition for better recall of hues characteristic of maximal spectral sensitivity of the three color receptors (Brown \& Wald, 1964), the opponent color pathways (Marks et al., 1964), the perceptually unique hues (Eichengren, 1976), or hues that are more readily named (Bornstein, 1973). This was also evident in Nilsson and Nelson's (1981) data. The results are consistent delay time are successively offset by $0.5 \mathrm{~nm}$ to avoid overlap. Increment standard errors of the average observer matches are shown as vertical lines at $575 \mathrm{~nm}$ as the mean of the standard errors across wavelengths for that delay time

with a random decay of the memory during the interval between the two stimuli.

The general similarity in Fig. 3 of the delayed matches to the hue difference-thresholds obtained by simultaneous matches is supported by their significant correlations. The memory difference-thresholds continued to resemble sensory difference-threshold functions when matches were delayed up to $14 \mathrm{~s}$. This is consistent with Nilsson and Nelson's data for similar memory times. Bae et al. (2015), Seliger (2002), and Uchikawa and Ikeda (1981) also found memory discrimination functions that resembled sensory functions though they only obtained these for memory times of $1 \mathrm{~s}$ or less. That this similarity occurs in the results for all six individual observers, as shown in Fig. 3 for CAS, and for Uchikawa and Ikeda's

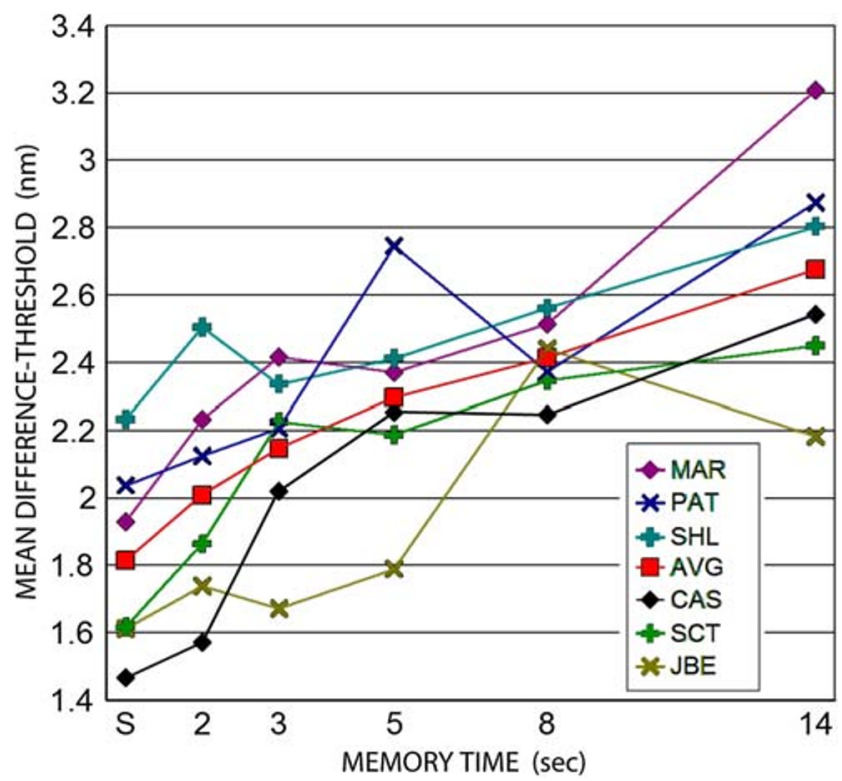

Fig. 4 How hue memory decayed over time. Hue difference-thresholds are averaged across wavelength as a function of memory time for the six observers and for their normalized average. The graphs are not offset 

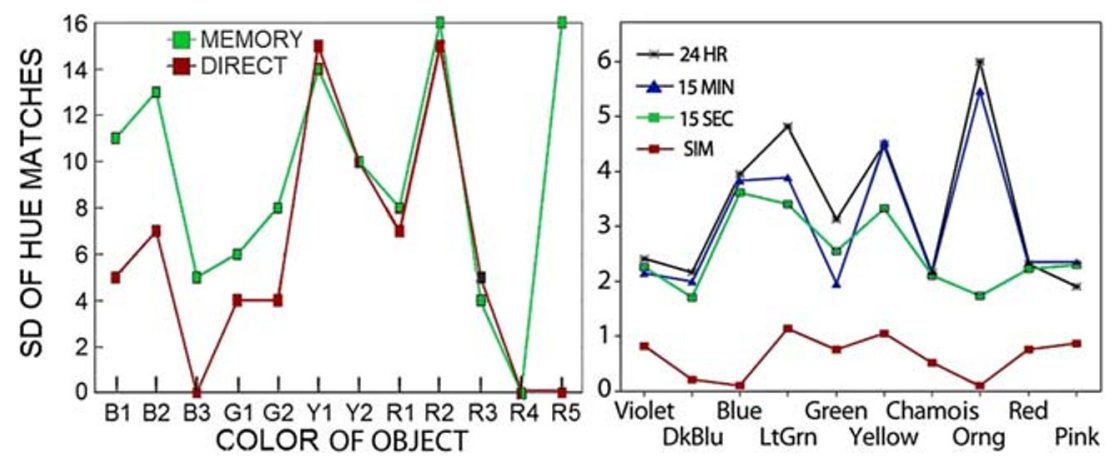

Fig. 5 Left: Bloj et al.'s (2016) standard deviations of Munsell hue matches are replotted as a function of the color of various objects. The objects are identified by their principle hue as being Blue, Green, Yellow, or Red. Right: Perez-Carpinell et al.'s (1998) standard deviations of hue

matches made simultaneously (SIM) and after delays of $15 \mathrm{~s}, 15 \mathrm{~min}$, and $24 \mathrm{~h}$ are replotted as a function of color. These functions have not been digitally filtered or offset to reduce overlap

three observers indicates that the similarity is not an artifact of averaging results across observers.

Different evidence that color memory discrimination is similar to sensory discrimination was found by replotting data from two studies that used Munsell chips to measure hue in terms of the CIE $1976 \mathrm{~L} * \mathrm{a} * \mathrm{~b} *$ color space. Bloj et al. (2016) had 12 subjects select chips to match the hue of 12 colored objects after viewing them for $30 \mathrm{~s}$ and later when viewing objects and chips simultaneously; Fig. 5 left shows their results. When data for R5 (the smallest and narrowest object) are ignored, the memory standard deviations correlate +0.85 with those obtained directly. Perez-Carpinell, Baldovi, de Fez, and Castro (1998) had 100 observers match the color of Munsell color chips when viewed simultaneously and after delays of $15 \mathrm{~s}, 15 \mathrm{~m}$, and $24 \mathrm{~h}$. Figure 5 right shows the hue standard deviations of their matches. The 15 -s discrimination function looks similar to the simultaneous function, though the +0.38 correlation is not significant (at 8 d.f., $95 \%$ confidence of similarity $=+0.63$ ). The weak similarity nevertheless provides some basis for comparison of measurements at longer memory times: at 15 min similarity drops substantially (correlation $=+0.15$ ), and little remains after $24 \mathrm{~h}$ (correlation $=$ +0.1 ). Their data suggest that some time between $15 \mathrm{~s}$ and 15 min memory discrimination changes. Further measurement of difference-thresholds at memory times in the 15-s to 15 min range might reveal a transition from short-term to longterm memory.

\section{Experiment 2: Dichoptic hue memory}

To see whether the resemblance of memory differencethresholds to sensory difference-thresholds was due to some type of retinal adaptation (e.g., Long \& O'Saben, 1989; Sakitt,

\footnotetext{
${ }^{1}$ This was prevented by UPEI dismantling the lab when the author contested mandatory retirement at age 65 years.
}

1975), the stimulus to be remembered was presented to one eye and the stimulus to be adjusted for a match presented to the other eye.

\section{Method}

Four observers made 18 matches of the hues produced by the same 20 wavelengths used in Experiment 1 when these were viewed simultaneously and after delays of 2, 3.5, 5, 6.5, 7.5, $8.5,10$, and $13 \mathrm{~s}$. The stimuli were presented with a dichoptic optical system using two Bausch \& Lomb Hi-Intensity monochromators with prisms to adjust interpupillary distance for a fused visual field similar to Fig. 1. One eye saw the upper slit, which presented the stimulus to be remembered. The other eye saw the lower slit, which contained the stimulus adjusted for a match.

\section{Results}

Dichoptic simultaneous and memory hue difference-threshold functions are shown in Fig. 6 for two observers, CAS and PHL. They generally resemble those obtained in Experiment 1 using monocular viewing. The average correlations of CAS's and PHL's memory difference-threshold functions to their sensory difference-threshold functions were +0.81 and +0.57 , respectively (at 18 d.f., $99 \%$ confidence $=0.56$ ). The average memory-to-sensory function correlations for the other two observers $(+0.49$ and +0.45$)$ were just significant at $95 \%$ confidence $(r>0.44)$. Based on the similar memory times used for monocular matches in Experiment 1, CAS's monocular and dichoptic difference-threshold functions on average correlated with $99 \%$ confidence at +0.62 .

Averaging these data across wavelength to see how the difference-thresholds generally changed with memory time produced different trends than the monocular data. The dichoptic wavelength-average difference-thresholds increase over time, but less and not as smoothly as the monocular 


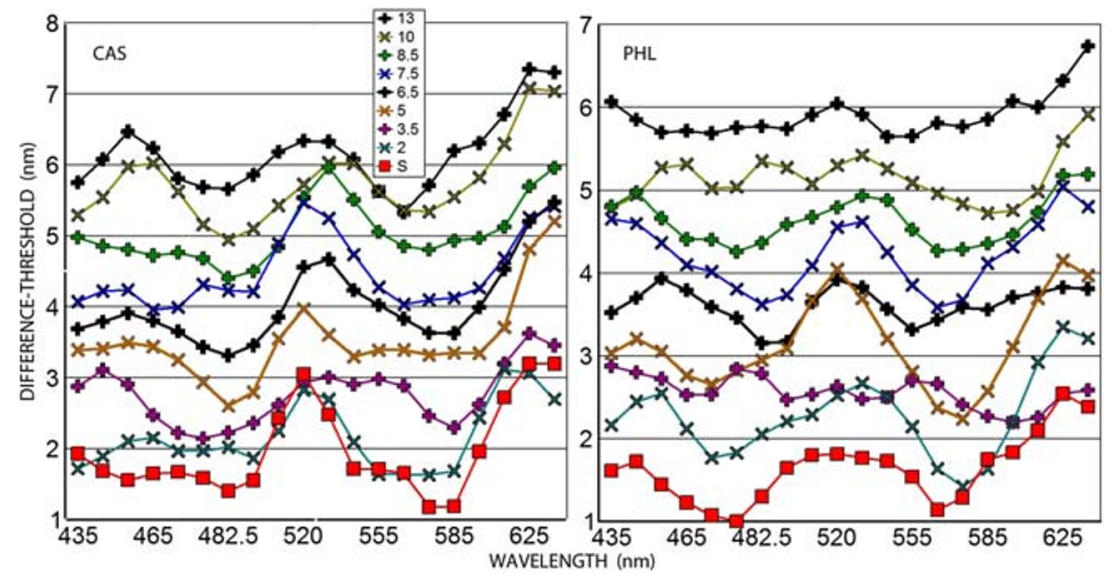

Fig. 6 Dichoptic measurement of hue memory. Wavelength differencethresholds are shown as a function of wavelength when matched simultaneously ( $\mathrm{S}$ ) and after delays of $2-13 \mathrm{~s}$ as indicated in the key. The stimulus to be remembered and the stimulus adjusted to match it were presented to different eyes. The delayed functions are successively offset

difference-thresholds; compare Fig. 7 with Fig. 4. The mean dichoptic difference-threshold, $2.1 \mathrm{~nm}$, at $13 \mathrm{~s}$ is notably less than the monocular difference-threshold, $2.7 \mathrm{~nm}$, at $14 \mathrm{~s}$. There is a small but consistent decrease in averaged difference-thresholds from the simultaneous matches to matches made at $3.5 \mathrm{~s}$ that is not evident in the monocular matches. Only after $3.5 \mathrm{~s}$ do the dichoptic matches begin to resemble a decay function. Yet the $0.13-\mathrm{nm}$ increase in difference-thresholds from 5 to $13 \mathrm{~s}$ is only about a third of the $0.33 \mathrm{~nm}$ increase in the monocular difference thresholds from 5 to $14 \mathrm{~s}$.

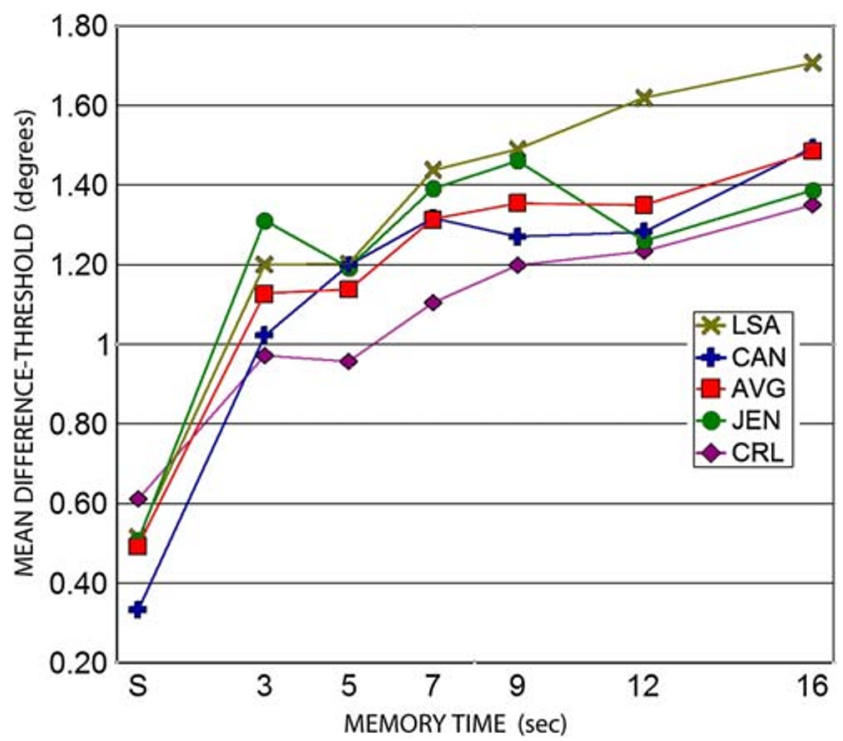

Fig. 7 How hue memory decays when measured dichoptically. The difference-thresholds for hue are shown as a function of memory time when averaged across wavelength for the four observers and their average. These functions are not offset to reduce overlap by $1 \mathrm{~nm}$ to eliminate overlaps. The left graph shows the results from observer CAS, whose monocular difference-difference thresholds are in Fig. 3. The right graph shows the results from observer PHL, who did not participate in Experiment 1

\section{Discussion}

The main purpose of the dichoptic measurements was to find out whether dichoptic memory difference-threshold functions resembled the monocular functions. They did. Except for differences in their rate of decay, they are similar. This indicates that similarity of memory matches to sensory matches is not attributable to some persisting retinal effect.

The decay of dichoptic difference-thresholds differs from the decay of monocular difference-thresholds in two aspects compare Fig. 7 with Fig. 4: One is the decrease in dichoptic difference-thresholds from successive to 3.5-s delayed matches. Caziot et al. (2015) report that stereopsis is complete at about one-third of a second, so latency does not explain the decrease. Based on my observations, it seems that attention to binocular fusion of the dichoptic stimuli at short delays between left and right eyes slightly distracted from the match judgments. The other difference is the slower increase in difference-thresholds as memory time extends beyond $3.5 \mathrm{~s}$. A study by Souza and Oberauer (2017) showed that central attention helps maintain information in working memory. This might explain the slower decay of the dichoptic memories as memory time increases beyond $3 \mathrm{~s}$. Clearly more research on memory of dichoptic stimuli is needed.

\section{Experiment 3: Line orientation memory}

Is the similarity of hue memory difference-thresholds to sensory difference-thresholds a unique feature of color vision? Line orientation is another visual stimulus with a complex difference-threshold function that would be discernable in memory measurements. Difference-thresholds are small for horizontal and vertical lines but substantially larger for lines 
at $45^{\circ}$ - the "oblique effect" (e.g., Andrews, 1967; Heeley \& Timney, 1988; Westheimer \& Beard, 1998). Like wavelength difference-thresholds, orientation difference-thresholds are clearly linked to sensory processing. Mansfield (1974) and Orban, Vandenbussche, and Vogels (1984) relate such results to variation in the receptive field distribution of cortical neurons sensitive to line orientation.

A few studies have measured memory for line orientation. Jastrow (1892) had observers reproduce from memory the orientation of lines at $15-165^{\circ}$ immediately after viewing them. Their errors were larger compared to errors when drawing the lines while viewing both lines. Though the memory time would have varied depending on the time taken to make the matches, the memory errors mirrored the simultaneous errors as a function of line angle. Magnussen, Landro, and Johnsen (1985) had subjects decide whether two lines had the same or different orientation when separated by $0.2,2$, 5.5, and $9.9 \mathrm{~s}$. Percent correct decisions decreased as memory time increased. They used lines at $0,30,60$, and $90^{\circ}$, but do not present the data at individual angles to show differencethreshold functions. Vogels and Orban (1986) also used a twoalternative forced-choice method to measure differencethresholds for a horizontal and an oblique line at five memory times from 0.65 to $10.4 \mathrm{~s}$. That the larger difference-thresholds for the oblique lines increased similarly with memory time as the smaller difference-thresholds for the horizontal lines is consistent with the hue memory data. Pertzov, Manohar, and Husain (2016) had observers match the orientation of bars at various orientations after $0.1-, 1-, 2-$, and 3-s delays. Their difference-thresholds increased with memory time, but data for individual angles are not reported. Other studies have used line orientation as an independent variable in memory experiments (e.g., Bae \& Luck, 2017; Zhang \& Luck, 2011), but the emphasis in these studies has been on other factors such as interference and quantitative effects. No study was found that reported difference-thresholds as a function of line angle at a memory time longer than Jastrow's.

\section{Method}

Four observers made 16 matches to each of 18 line orientations (from $0^{\circ}$ to $85^{\circ}$ in $5^{\circ}$-steps) when the lines were presented successively and after delays of 3, 5, 7, 9, 12, and $16 \mathrm{~s}$. A Scientific Prototype 800-F tachistoscope was modified by placing a rotatable black wire $\left(3.8^{\circ}\right.$ long, $7^{\prime}$ wide) at the image plane in each channel. The orientation of one wire was adjusted by the observer. Orientation of the second wire was set by a computer that also read the observer's orientation setting of the adjusted wire, and triggered lamp onsets at the above delays. Illumination durations of $1 \mathrm{~s}$ (rise and decay time about $40 \mathrm{~ms}$ ) were controlled by the tachistoscope. At the $30-\mathrm{cm}$ viewing distance, this produced a round $3.8^{\circ}$ visual field having a white background of $200 \mathrm{~cd} / \mathrm{m}^{2}$. A dim spot of light in the center of one wire helped the observer maintain fixation when neither channel was illuminated. The observer triggered the computer when to start each measurement and to record the angle of the adjusted line when a match was obtained.

\section{Results}

To see whether storing the image of a line shifted its recalled orientation, the angle to be recalled was subtracted from the mean angle of the 16 matches made successively and after the six delays. The results from observer LSA for each of the 18 line orientations are shown in Fig. 8. Average magnitude of the shifts across orientation angle equaled $0.25^{\circ}$ for the successive matches versus about $1^{\circ}$ for the delayed matches as noted by the numbers on the left of each function. Comparing the means of delayed matches with the means of successive matches as a function of orientation showed that these correlated by -0.06 (at 16 d.f., $95 \%$ confidence for " $r$ " $=0.47$ ) on the average across the delay times. LSA's overall mean matches had a mean correlation of -0.02 (at 124 d.f., $95 \%$ confidence $=0.18$ ) with those of the other three observers. This lack of consistency in orientation shifts at the various memory times indicates there was no systematic shift in memory of the line orientations.

Figure 9 shows the orientation difference-thresholds as a function of line orientation when matched successively and after delays of 3-16 s for observer LSA and the normalized average of the four observers. All observers' memory difference-thresholds tended to be maximal around $45^{\circ}$ as memory time increased. However, the maximum sensory difference-thresholds varied substantially amongst the

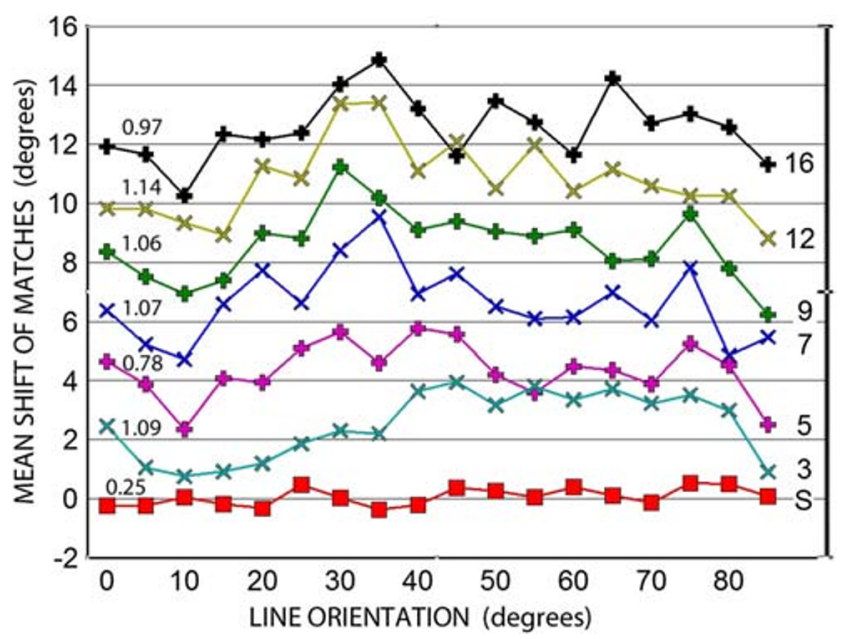

Fig. 8 Mean difference in angle of observer LSA's 16 line orientation matches from the angle of the other line, as a function of line orientation when made successively (S) and after delays of 3, 5, 7, 9, 12, and $16 \mathrm{~s}$ as labeled along the right side of the graph. The delayed functions are successively offset by $2^{\circ}$ to prevent overlap. The numbers on the left end of each function are the average magnitudes of the difference in angle between the angle to be matched and the observer's match averaged across all orientations for that memory time 

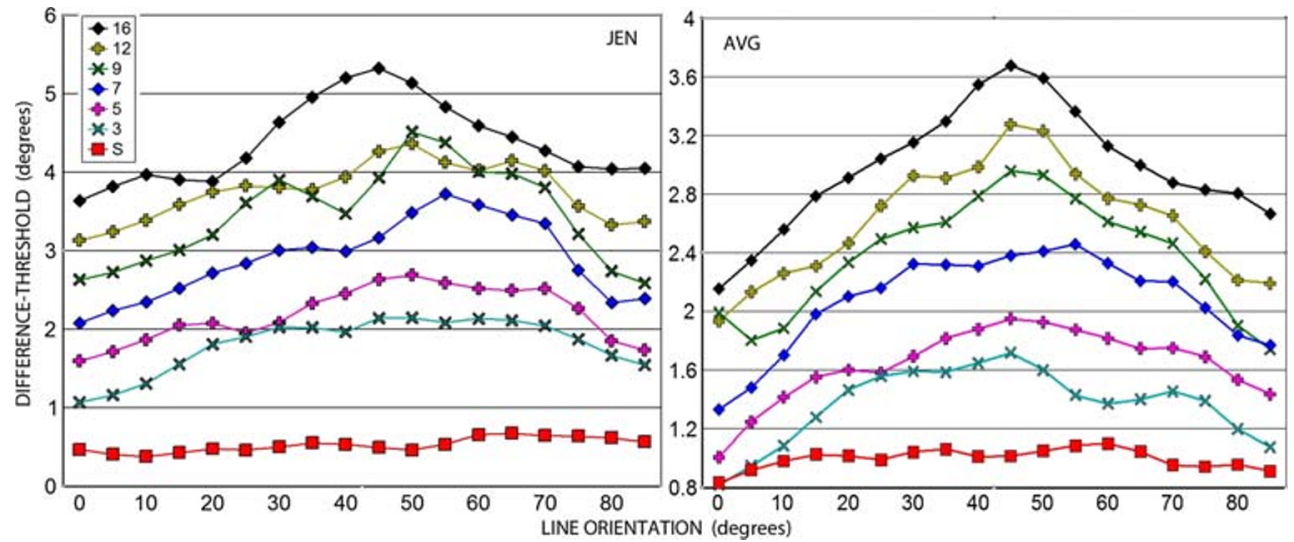

Fig. 9 How orientation angle and time in memory affected the accuracy of matching the orientation of a line. These difference-thresholds were measured as a function of line angle when matched successively (S) and after delays of from $3 \mathrm{~s}$ to $16 \mathrm{~s}$ by observer LSA and the average of

observers. When similarity of the memory differencethreshold functions to the sensory was measured in terms of correlation, only the average observer functions and those of observer CLY were significant (mean $\mathrm{r}=+0.78$ and +0.61 respectively, at 16 d.f., $95 \%$ confidence $=0.47$ ). This lack of similarity of the memory to sensory functions for three of the observers seems due to the indistinctness of their sensory functions as evident for LSA in Fig. 9. It is likely that the mean shift of $0.33^{\circ}$ in the sensory matches across angles and observers approached the instrumental limit of measurement error. LSA's difference-thresholds in Fig. 9 show that as memory time increased, the longer memory functions maintained the characteristic shape of the 3-s memory function. This was the case for all observers. The mean similarity of the observers' longer memory functions to their 3-s function was a significant +0.76 (at 16 d.f., $99 \%$ confidence $=0.59$ ).

To see whether there was a general trend in how accuracy of the orientation matches changed over time, the differencethresholds were averaged across the angles and plotted for each observer and for the average observer as a function of time in memory (Fig. 10). These functions more clearly suggest an exponential decay than did the hue decay functions shown in Fig. 4. Relative to the mean difference-threshold for successive matches, the observer-averaged memory difference-thresholds were doubled by the 3 -s memory time.

\section{Discussion}

The main purpose of this experiment was to find out whether similarity of memory difference-thresholds to sensory difference-thresholds was an effect unique to color. Would it also occur with memory for a stimulus that involved a spatial feature? The results in Fig. 9 clearly show that this also happens with the memory for line orientation. The sensory difference-threshold functions for three observers were not sufficiently defined due to their small size relative to matches of the four observers. The delayed matching functions are successively offset by $0.5^{\circ}$. The successive functions have been amplified by 2 to show their shape

measurement limits. The memory difference-thresholds are consistent with previous results obtained by various methods: orientation matching (Chapanis, 1951), detection (Ogilvie \& Taylor, 1958), and discrimination (Foster \& Westland, 1995). Jastrow (1892) and Vogels and Orban (1986) also measured how well line orientation could be remembered. Jastrow's memory measurements of lines at angles from $15^{\circ}$ to $165^{\circ}$ in $15^{\circ}$ steps were based on participants drawing a line from memory immediately after seeing a line at a certain angle. This would involve a memory that was only a few seconds old. Vogels and Orban (1986) only used horizontal and oblique lines, but measured memory after several more defined delays $(0.65,1.3,2.6,5.2$, and $10.4 \mathrm{~s})$ using a two-position forcedchoice procedure. Despite these differences in method, both studies also show that the discrimination of line angle from memory is similar to the sensory discrimination of line angle.

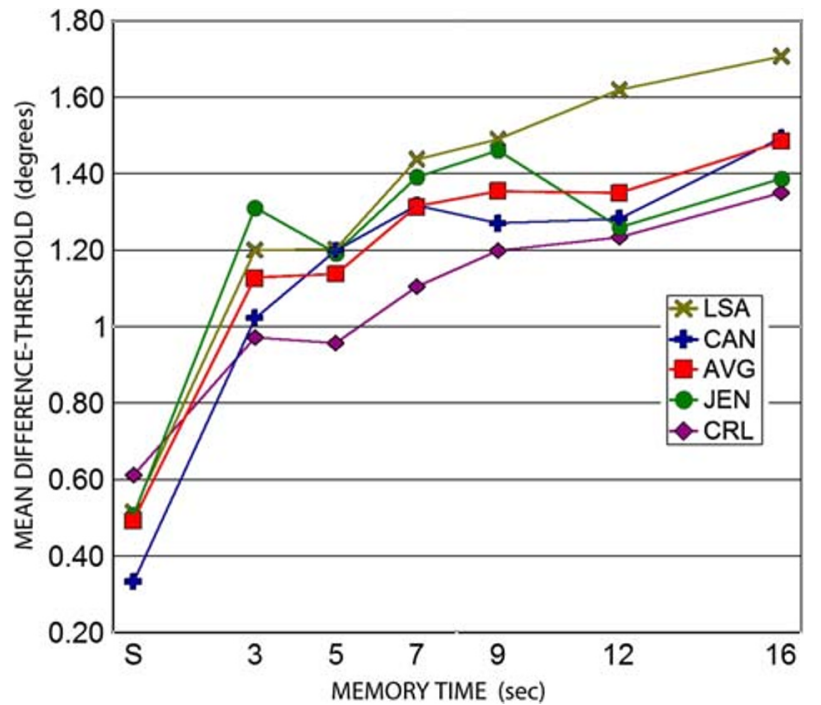

Fig. 10 Decay in accuracy of line orientation memory as a function of time. The line orientation difference-thresholds are averaged across the orientation angles for the four observers and their average 
Experiments 1 and 2 found no tendency for hue matches to systematically drift over time in memory; Fig. 8 shows that neither did the line orientation matches. Chapanis (1951) and Jastrow (1892) reported similar results. There is no evidence that orientation memory is biased towards certain orientations in the absence of other context. Since these measurements were made in a dim sound-deadened booth, random visual noise is a likely source of the variability that was largely cancelled out by averaging the matches. Figure 10 indicates that the half-life of line orientation memory was about $3 \mathrm{~s}$. This is substantially shorter than the half-life of hue memory indicated by the results in Experiments 1 and 2 to exceed $14 \mathrm{~s}$ and by Nilsson and Nelson's (1981) estimate of $24 \mathrm{~s}$.

\section{Experiment 4: Line-orientation memory by forced-choice}

The question remained whether the similarity of memory difference-thresholds was somehow an artifact of using a matching method to measure the memory difference-thresholds. Forced-choice methods (e.g., Foster \& Westland, 1995; Heeley \& Timney 1988; Orban, Vandenbussche \& Vogels, 1984; Westheimer \& Beard,1998) produced difference-threshold functions that are similar to those obtained by studies that also used a matching method (Andrews, 1967; Chapanis, 1951). However, the only known study that had measured complete memory difference-thresholds as a function of a complete set of orientation angles from $0^{\circ}$ to $90^{\circ}$, Jastrow (1892), also used a matching method. Therefore it was possible that the similarity of the memory functions to sensory functions only occurred when measured by an orientation-matching method. The computerized apparatus for measuring line orientation memory enabled

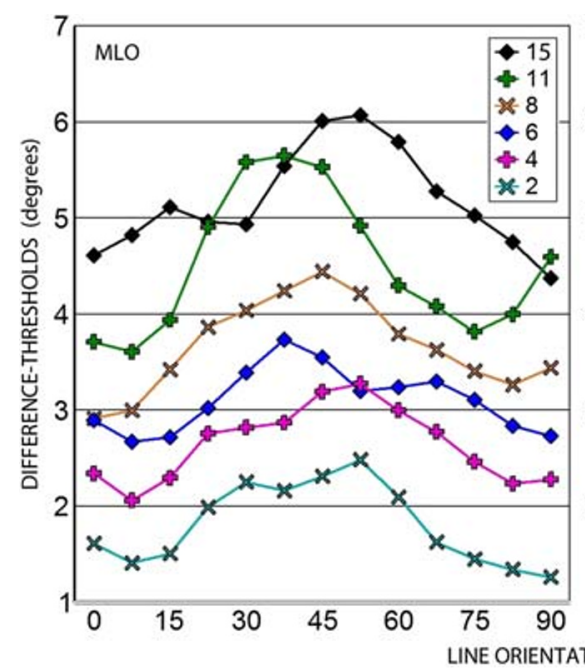

Fig. 11 Memory difference-thresholds measured by the forced-choice method are shown as a function of line orientation angle after delays of $2-15 \mathrm{~s}$ between the stimuli. The functions have been offset by $0.5^{\circ}$ to measuring orientation difference-thresholds with enough speed to make a forced-choice method possible.

\section{Method}

The main purpose of this experiment was to determine if the resemblance of memory difference-threshold functions to sensory functions was somehow a result of using the psychophysical method of matching to make the measurements. To use a forced-choice method to measure the difference-thresholds, two temporal positions were used. Memory functions were measured with delays of $2,4,6,8,11$, and $15 \mathrm{~s}$ between the two positions. The observer's task was to decide which position (first or second) had the line with the steeper orientation a procedure similar to Heeley and Timney's (1988). To reduce the number of measurements required by the forced-choice method to 7,800 per observer, a sensory difference-threshold function obtainable by successive comparison was not measured. Four observers made 20 choices at each of five angular offsets from each of 13 base orientation angles $(0,7.5,15, \ldots$, $90^{\circ}$ ). Difference-thresholds at $75 \%$ correct choice of the steeper line were estimated by linear interpolation. The orders of presentation were counterbalanced and pseudo-randomized to ensure that successive offsets and base angles did not repeat in short order.

\section{Results and discussion}

Figure 11 shows the orientation memory difference-thresholds as a function of orientation angle for each of the six delay times from $2 \mathrm{~s}$ to $15 \mathrm{~s}$ from one observer, MLO, and the average results from all four observers. Both graphs show that the memory difference-thresholds do not flatten or become less distinct as memory time increased. They continue to

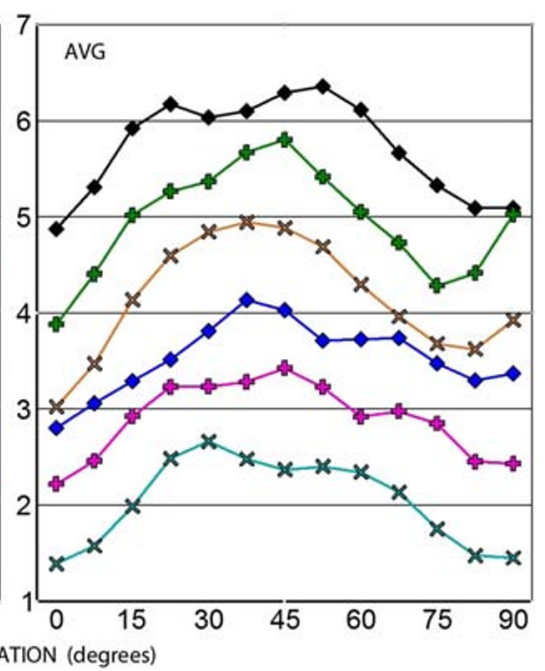

minimize overlap. On the left are the results from observer MLO; on the right are the average results of the four observers 
resemble the sensory functions found in other studies using forced-choice methods (e.g., Foster \& Westland, 1995; Heely \& Timney 1988; Westheimer \& Beard 1998) as well as those using matching (e.g., Andrews, 1967; Chapanis, 1951; Jastrow, 1892) and the successively matched results in Experiment 3 (Fig. 9). Extrapolating across the orientations used for the sensory difference-thresholds in Experiment 3 enabled estimating difference-thresholds at the same angles (except $90^{\circ}$ ) as those used for forced-choice. The average observer forced-choice memory functions had a mean correlation of $+0.78(95 \%$ confidence at 10 d.f. $=0.58)$ with the average observer sensory function in Experiment 3 . Similarity of the forced-choice memory functions to sensory functions was not an artifact of using the matching method to measure difference-thresholds.

\section{Experiment 5: Grating orientation memory}

The line orientation difference-threshold functions obtained from memory were less similar to their sensory counterparts, and differences between observers were larger than was the case for hue memory. Large observer differences in orientation acuity were also noted by Heeley and Timney (1988). This might be partly attributed to the image of a 7-min visual-angle wide line on the retina being more susceptible to eye movement and local astigmatic irregularities than the image of a 30-min wide monochromator slit. Gratings would produce a wider retinal image that could still be tested for differencethresholds as a function of angle. Though observers in Experiments 3 and 4 were screened for normal acuity, the independence of orientation discrimination from spatial frequency (Bradley \& Skottun, 1984; Burbeck \& Regan, 1983) might also reduce observer variability from minor differences and changes in acuity. Grating orientation differencethresholds vary as a function of angle in a similar complex manner as do those for single lines (e.g., Campbell, Kulikowski, \& Levinson, 1966; Heeley \& Timney, 1988). Therefore similarity of their memory difference-threshold function to a sensory function would be discernible. They are also similarly related to sensory processing by cortical receptive fields (e.g., Campbell \& Kulikowski, 1966; Furmanski \& Engel, 2000). Would greater consistency of the memory results within and between observers be found by using gratings?

Magnussen, Idas, and Myhre (1998), Rademaker et al., (2018), and Shin, Zou, and Ma (2017) have found that the precision of memory for grating orientation decreases as memory times increase up to 5,6 , and $12 \mathrm{~s}$, respectively. However, they do not show how accuracy varied as a function of orientation angle. Pratte, Park, Rademaker, and Tong (2017) had observers match the orientation of gratings after $3 \mathrm{~s}$. Their delayed difference-threshold function looks like a sensory difference-threshold function, though they did not measure the sensory function. Balikou et al. (2015) had observers match grating orientations that varied randomly in $1^{\circ}$ steps from $0^{\circ}$ to $180^{\circ}$ after delays of $0.5-4.5 \mathrm{~s}$. A sinusoidal model was fitted to the resulting scatter plot of error as a function of orientation. This model revealed a series of perfectly uniform sensitivity functions even as sensitivity decreased with increasing memory time. Unfortunately, memory time in both Pratte et al.'s and Balikou's experiments was not well defined. When presented with a probe up to $4.5 \mathrm{~s}$ after seeing the grating to be remembered, their observers would have taken varying amounts of time to make their matches. Previous research does not indicate whether more general insight into what comes out of memory may be gained by measuring how grating orientation difference-threshold functions vary over memory times longer than a few seconds.

\section{Method}

Six observers made eight iterative matches of grating orientation for 13 orientations from $1,7.5,15, \ldots$, to $90^{\circ}$ when viewing a pair of 1-s duration gratings successively and after delays of 2-24 s. These sinusoidal luminance-modulated gratings were generated on a Tektronix 608 cathode ray monitor with a fast P31 phosphor by a Picasso analog computer controlled through digital-to-analog converters by a digital computer. A $15^{\circ}$ visual angle white-background field of view was maintained at a steady $9 \mathrm{~cd} / \mathrm{m}^{2}$ luminance. Within this field, either of two $6^{\circ}$ bulls-eye modulated fields could be presented. These fields contained about seven grating cycles whose luminance varied between 12 and $7 \mathrm{~cd} / \mathrm{m}^{2}$. The screen was binocularly viewed from a distance of $37.5 \mathrm{~cm}$. Figure 12 illustrates the viewing conditions.

\section{Results}

The sensory and memory difference-thresholds for grating orientation as a function of orientation angle obtained by matching after delays from $0 \mathrm{~s}$ to $24 \mathrm{~s}$ are shown in Fig. 13

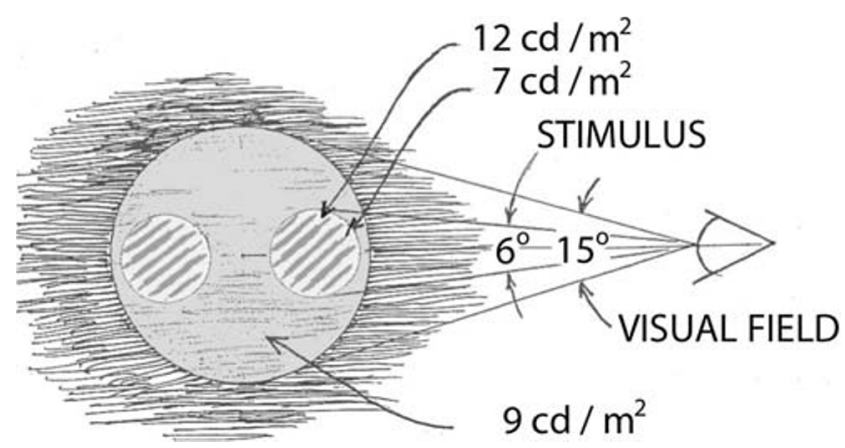

Fig. 12 Sketch of how the two grating stimuli would have appeared if viewed simultaneously. The observer adjusted the orientation angle of the right grating to match the left 


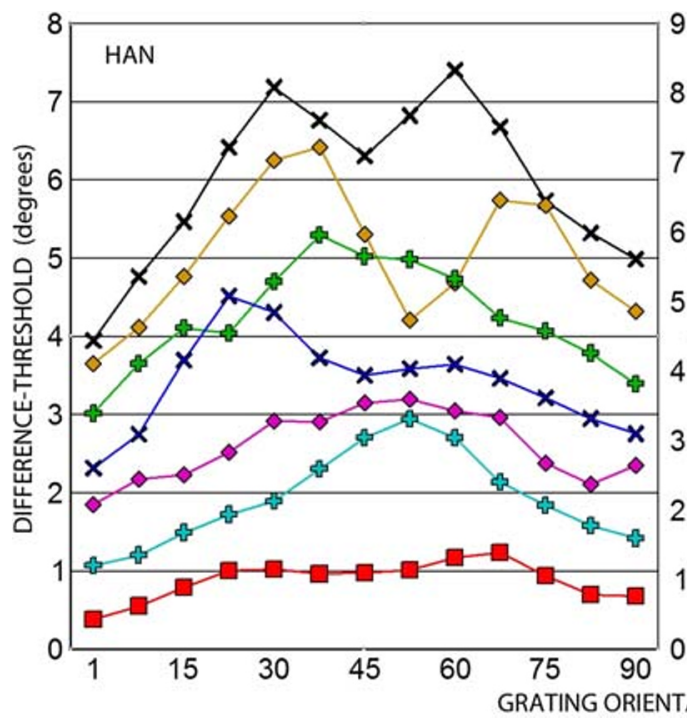

Fig. 13 Difference-thresholds for grating orientation. These are shown as a function of grating angle obtained by successive matching (S) and after delays of 2 to $24 \mathrm{~s}$. The functions are successively offset by $0.25^{\circ}$. The left graph shows the results for observer HAN. The right graph shows the

for observer HAN and the average of all six observers. These functions show an oblique effect that persists in memory like those for line orientation memory in Experiments 3 and 4. The similarity of each observer's memory difference-threshold functions to their sensory difference-threshold function was measured in terms of their correlations. See Table 1. Observer HAN had the highest mean correlation at +0.82 , while mean correlation of the average observer functions in Fig. 13 was +0.94 (at $11^{\circ}$ d.f. a correlation must exceed 0.68 for $99 \%$ confidence that the similarity was not due to chance). Gratings evidently did reduce intra-observer variability compared to the results for line orientation memory, though differences between observers remained.

Table 1 Correlations of each of the six 2- to 24-s delayed, memory difference-threshold functions with their successively matched, sensory difference-threshold functions as a function of 13 grating orientations from $1^{\circ}$ to $90^{\circ}$ for each of the six observers, and correlation of their average

\begin{tabular}{llllllll}
\hline \multirow{2}{*}{ Memory } & \multicolumn{2}{l}{ Observers } & & & & & Avg \\
\cline { 2 - 6 } Time & HAN & PWB & NBU & MMP & KUB & ALD & Obs \\
\hline 2 & 0.78 & 0.72 & 0.93 & 0.79 & 0.66 & 0.80 & 0.93 \\
4 & 0.85 & 0.66 & 0.51 & 0.68 & 0.40 & 0.78 & 0.80 \\
8 & 0.74 & 0.74 & 0.73 & 0.33 & 0.49 & 0.73 & 0.89 \\
12 & 0.75 & 0.76 & 0.64 & 0.68 & -0.21 & 0.63 & 0.91 \\
16 & 0.66 & 0.81 & 0.81 & 0.49 & 0.16 & 0.71 & 0.93 \\
24 & 0.94 & 0.79 & 0.83 & 0.71 & 0.39 & 0.81 & 0.95 \\
Mean & 0.79 & 0.75 & 0.74 & 0.61 & 0.32 & 0.74 & 0.90 \\
\hline
\end{tabular}

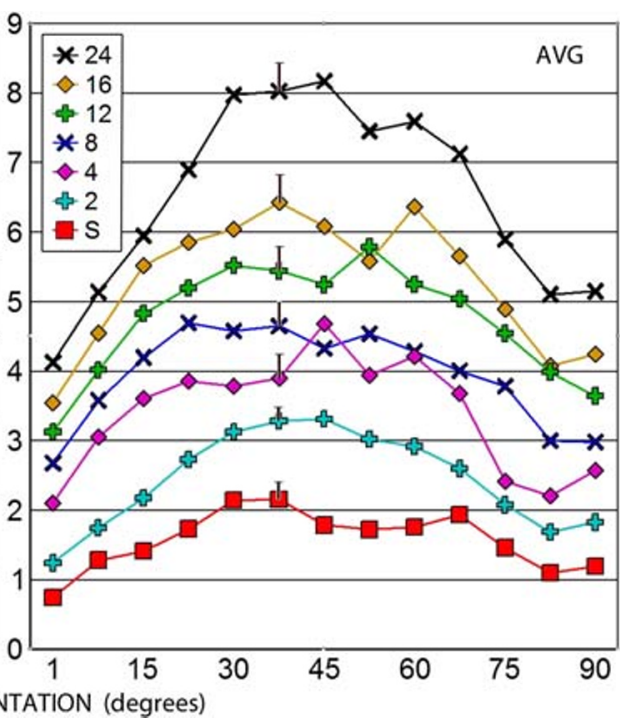

average results from all six observers. Vertical bars at the $37.5^{\circ}$ data point in each function represent the mean increment standard error for that function

The grating difference-thresholds tended to be nearly twice as large as those for line orientation. This size difference is more evident by comparing the decay functions obtained by averaging the difference-thresholds across orientation angles shown in Fig. 14 with the decay functions for line orientation in Fig. 10. Evidently the orientation of diffuse sinusoidal grating features was more difficult to discern than the orientation of sharp lines. The average sensory grating difference-threshold was about $1.5^{\circ}$ compared to $0.5^{\circ}$ in Experiment 3 . By about $18 \mathrm{~s}$ (extrapolated), the difference-thresholds doubled in magnitude. This half-life estimate was longer than the 3-s estimate for line-orientation memory (Fig. 10) and shorter

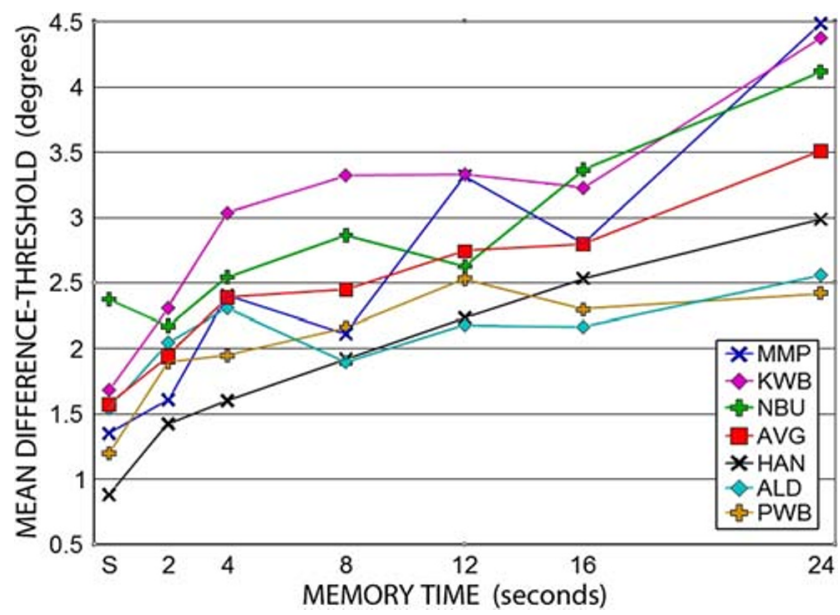

Fig. 14 How accuracy of the grating orientation matches decayed in memory. Grating orientation difference-thresholds are averaged across orientation angle as a function of memory time for the six observers 
than the indication in Fig. 4 that the half-life of hue memory was substantially longer than $14 \mathrm{~s}$.

\section{Discussion}

Plotted as a function of grating orientation angle, the difference-thresholds at memory times up to $24 \mathrm{~s}$ were similar to the sensory difference-thresholds. Averaged across the six observers, the present series of memory functions at various times from $2 \mathrm{~s}$ to $24 \mathrm{~s}$ were sufficiently similar to the average sensory function to correlate +0.90 . This is not as similar as the apparently perfect similarity of Balikou et al.'s (2015) modeled series of functions at 2.5, 3.5, and $4.5 \mathrm{~s}$ to their 0.5 $\mathrm{s}$ function. However, their functions were derived by modeling and are not direct data. Consistent with this effect are Pratte et al.'s (2017) orientation discrimination functions measured by matches made after a 3 -s memory time. Whether they involved sets of 1,2, or 3 gratings, those functions are similar to sensory discrimination functions. A comparable effect is evident in Lee and Harris' (1996) grating contrast difference-thresholds measured at memory times of 1,3 , and $10 \mathrm{~s}$. Plotted as a function of spatial frequency, their U-shaped difference-threshold functions at $3 \mathrm{~s}$ and $10 \mathrm{~s}$ are similar to their 1-s function.

The different memory half-lives found for hue ( $>14 \mathrm{~s}$ from the present study or about $24 \mathrm{~s}$ based on Nilsson and Nelson, 1981), line orientation (3 s), and grating orientation (18 s) indicate that these different characteristics of visual stimuli decayed at different rate (see Figs. 4, 10, and 14). Rademaker et al. (2018) also found that grating orientation memory decayed faster than color memory. Becker, Miller, and Liu (2013) suggest that a greater robustness of color memory compared to orientation memory is attributable to the greater richness of the color repertoire, which could be interpreted as containing more information than orientation.

Other examples of different decay rates for various visual stimuli include: contrast, spatial frequency, movement speed, and movement direction memory (Pasternak \& Greenlee, 2005); faces (Rademaker et al., 2018); line length (Hegelmaier, 1852 - cited by Laming \& Laming, 1992), and line curvature (Sakai, 2003). Yet some studies have found that certain visual memories such as spatial frequency of gratings (e.g., Magnussen, 2009 ) and complex shapes (Mercer, 2014) undergo little or no decay. Furthermore, it seems that conclusions about the decay of visual memory depend on the type of feature, the accuracy of measuring changes in memory, how long the memory is held, and what criteria are used. For example, Fahle and Harris (1992) conclude that memory for vernier offset is "fairly robust" after up to $8 \mathrm{~s}$, which is about the acuity half-life of their data, and Hole (1996) concludes that spatial discrimination was still "tolerably" accurate after thresholds discriminating the gap between two points in an image had doubled at delays of $30 \mathrm{~s}$ because this was less than
$14 \%$ of the distance between the points. Since sensory processing differs substantially for the above visual features, resolving such issues could improve understanding of how various types of sensory information are stored.

\section{Modeling memory matches}

Experiments 1-5 show that when simple visual features of color or linear orientation are matched from memory, the difference-thresholds for those features are similar to the difference-thresholds when those features are matched by direct observation. It is as though memory matches are obtained by a comparison of two sets of neural activity that are similar to the two sets of neural activity being compared during direct observation. This would actually be the case if the activity retrieved from memory resembled the sensory response. Yet the data also show that the accuracy of the memory matches decays with memory time.

Forgetting may be due to passive decay (e.g., Brown, 1958; Ricker, Spiegel, \& Cowan, 2014) or due to interference by further information (e.g., Melton, 1963; Souza \& Oberauer, 2015), or some other process (e.g., Mercer, 2014). In the above Experiments 1-5, observers sat in darkness with their heads held steady by a bite-board system. There was no other appreciable source of visual information that might interfere with whatever storage process occurred between seeing the "test" and seeing the "matching" stimuli. Therefore, it seems reasonable to assume that the loss in accuracy of their matches was due to decay of the stored information (Baddeley \& Scott, 1971). Whether that decay involved a growing variability arising from some maintenance process, a disintegration of neural circuits or molecular structures that encode the information, or an accumulation of noise (e.g., Amtul \& Rahman, 2016; Sreenivasan, Curtis, \& D’Esposito, 2014), the general consequence is that retrieved information becomes less distinct with time.

The minimal systematic shift in the averages of many repeated matches over increasing memory times (Figs. 2 and 8) is consistent with random influences on each match. As one matches a hue perceptually or from memory, some matches are made to slightly longer and some to slightly shorter wavelengths. These tend to balance out when averaged. So even though the memories become less distinct and the individual matches more variable, the averaged matches change little, giving the impression that the memories remain accurate. That is not what happens with memory difference-thresholds. As memory becomes less distinct, larger differences from the original stimulus become acceptable as a match, the matches become more variable, and the difference-thresholds increase. It is the second-moment difference-thresholds, not firstmoment averages, that reflect memory decay.

As reviewed in the Introduction, the distinct shape of sensory difference-thresholds as a function of stimulus 
level arises from variation in sensory response to the levels. Nilsson and Nelson (1981), Perez-Carpinell et al. (1998), Seliger (2002), and Experiments 1-5 above show that similar difference-threshold functions arise from memory. Evidently these memory functions reflect similar variation in memory responses at those levels. The simplest explanation for this would be that the memory responses resembled the sensory responses at those levels. Yet those same data also showed that memory accuracy decayed over time. How could memory responses have retained their distinctness despite the decay?

The answer may lie with the repetitive nature of the difference-threshold measurements. Averaging is one of the oldest methods of extracting signal from noise (e.g., Dawson, 1954; Regan, 2009). The difference-thresholds are based on the second statistical moment - standard deviation of repeated matches from their average at each level. Perhaps the averaging inherent in standard deviations extracts a sensorylike response in memory from the noise that produces memory decay.

\section{Method}

To test this possibility, a neural network model of stimuli being matched from memory was built in a QuattroPro spreadsheet (Macho, 2002). It is based on recognition by correlation between the memory trace of a stimulus and the sensory responses to an array of stimuli similar to and including the remembered stimulus. This simulates the iterative matching procedure used by the observers in Experiments 1, 2,3 , and 5 . Recognition by correlation has been widely used in memory models (e.g., Dara, Satyanarayana, \& Govardhan, 2014; Metcalfe, 1991; Mewhort \& Johns, 2005; Pribram et al., 1974). Neural correlates of similarity computations have been observed in the visual cortex (e.g., Mikhailova et al., 2017; van Vugt, Sekuler, Wilson, \& Kahana, 2013). Austin and Lees (2000) show how recognition operations can be rapidly performed by structurally simple correlation matrices. To explain how the model works, a simplified version is illustrated in Fig. 15.

In the upper left of Fig. 15, a certain STIMULUS TO REMEMBER is identified by a diagonal series of four letters (A, B, C, \& D), which represent a four-part SENSORY RESPONSE pattern to that stimulus. Each part is a random number. That response passes through a SENSORY BUFFER to the right and becomes a series of four-part MEMORY TRACES at various memory times further to the right. The value of each memory part undergoes a 16-"second" half-life decay of the sensory response elements in memory: In the first MEMORY TRACE at $2 \mathrm{~s}$, each of those memory elements has $80 \%$ of the value of each stimulus element and $20 \%$ the value of a random number (e.g., $0.8 * \mathrm{~A}+0.2$ "rand", $0.8 * \mathrm{~B}$ $+0.2 *$ "rand" value, etc). The "rand" is a QuattroPro function that produces a random number between 0 and 1 . By $16 \mathrm{~s}$ each element of the memory trace contains only half the value of the sensory response and the other half is random $(0.5 * \mathrm{~A}+$ $0.5 *$ "rand", etc). These memory traces are compared with a series of stimuli that are similar to the STIMULUS TO REMEMBER and selects the one with the highest correlation as a match.

The comparison stimuli are created as follows: A difference (diff) from the STIMULUS TO REMEMBER is produced using a fractional value of each element in its SENSORY RESPONSE. In the center left, a vertical series of COMPARISON STIMULI is prepared by adding or subtracting 1 to five diffs to or from each element in that row of the original SENSORY RESPONSE. In the middle of that set is a row of parts that replicate the response to the STIMULUS TO REMEMBER.

In the center of Fig. 15 is a correlation matrix where each COMPARISON STIMULUS RESPONSE is compared to the MEMORY TRACE at each memory time. (The actual calculations are done off-screen.) For each memory time, the diff value of the COMPARISON STIMULUS having the highest correlation with the MEMORY TRACE is added to the corresponding column of MATCHES below. From those columns are calculated the mean and standard deviation of the MATCHES at each memory time.

To obtain matches from a series of stimuli that correspond to different levels such as wavelengths or orientations, these calculations are repeated with several more STIMULI TO REMEMBER represented by a new four-part series of random numbers. To simulate variations in sensitivity to these levels, their random number range is changed. A greater range tends to result in larger differences between the comparison stimuli. Therefore matches that are closer to the STIMULUS TO REMEMBER will likely be the best match. This results in smaller standard deviations of the matches. Using the standard deviations as difference-thresholds, the program displays the difference-thresholds as a function of stimulus level for each memory time.

As described so far, the simplified model does not provide a realistic simulation. Too frequently it selects the replicated STIMULUS TO REMEMBER as the best match. To make the matching task more difficult, additional randomness was added. Based partly on Wickelgren's (1974) suggestions: (1) a "memory-noisegain" factor was added to the decay calculations, (2) a "memory-gain" factor was added to the decay and the correlation calculations, and (3) a "transducer-noise" factor was added to calculate the comparison stimuli. It may seem counterintuitive, but a similar non-intuitive effect is demonstrated by Ermentrout, Galan, and Urban's (2008) finding that noise can make neural models more responsive, and by Zhang's (2008) proposal that random activity promotes memory consolidation. 


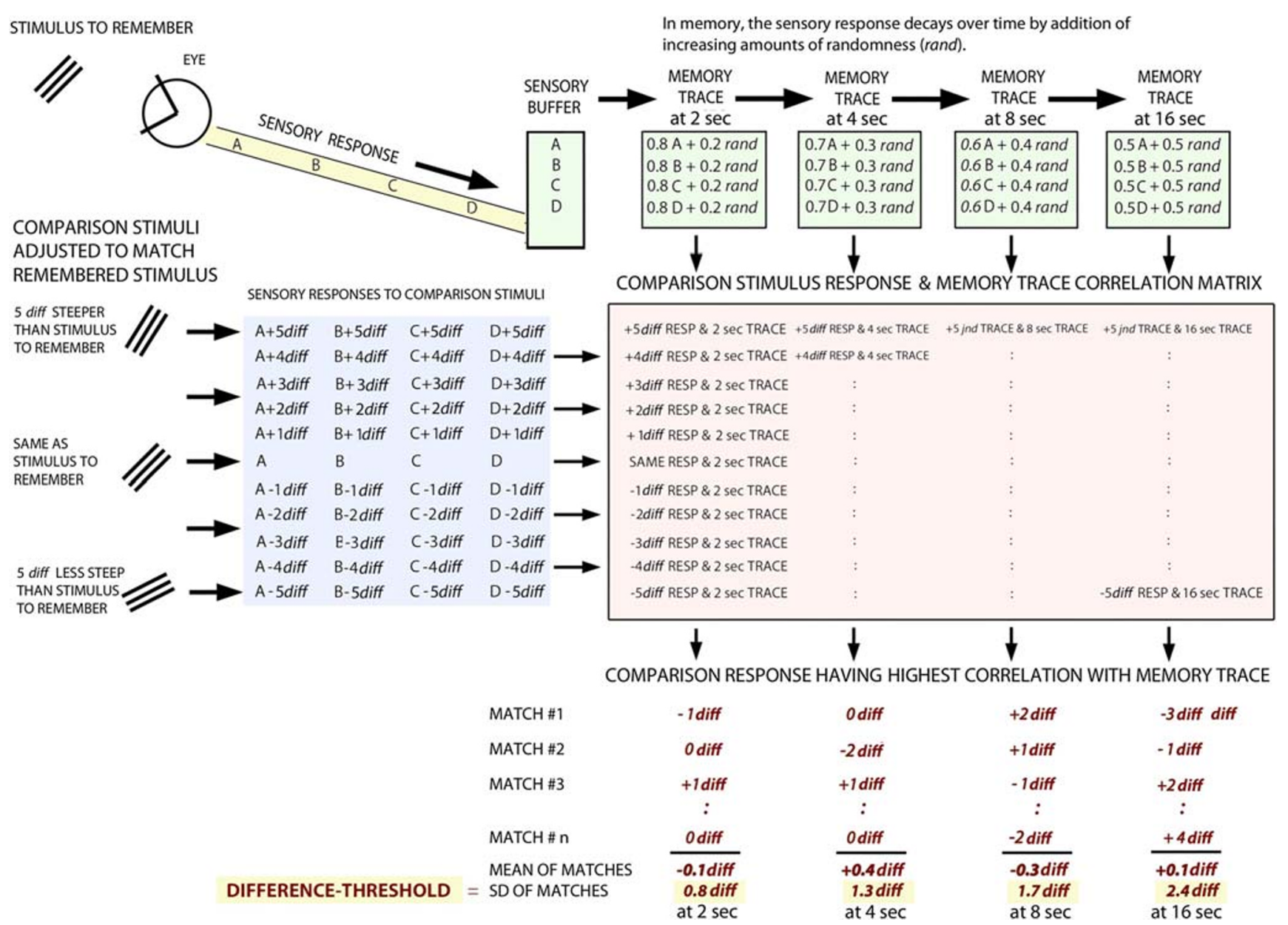

Fig. 15 A simplified diagram to explain how the QuattroPro spreadsheet model compares memory traces of a certain stimulus with a set of stimuli that vary in similarity to that certain stimulus. It takes the difference (diff) value of the comparison stimulus with the highest correlation to the trace as a "match." The match diffs are stored below the CORRELATION

In addition to more noise, the complete model uses a 13part SENSORY RESPONSE, 17 steps (+/- 8 diff) of COMPARISON STIMULI, and 16 stimulus levels. Difference-threshold functions were produced for simultaneous matches without memory decay and for seven simulated delayed matches from $2 \mathrm{~s}$ to $128 \mathrm{~s}$. To simulate decay in the memory traces, an increasing proportion of each trace part is replaced by a random value as the time increases. To simulate a half-life of $16 \mathrm{~s}$, the progressive decay rate was set so that $50 \%$ of the original STIMULUS TO REMEMBER remained at that time. At delays of $128 \mathrm{~s}$, only $17 \%$ of the SENSORY RESPONSE remains in the memory trace. Further details about the model are provided in the Appendix. The program is freely available from the author as a QuattoPro spreadsheet and also accessible from the Open Science Framework. For the following results, the model was extended to repeat the difference-threshold measurements for eight observers matching the same stimuli at each level. This enabled the model to produce memory difference-threshold functions for individual observers and for an average of their functions comparable to the experimental results in Figs. 3, 6, 9, and 13.

\section{Results and discussion}

The only reason to suppose that a model like this might produce memory difference-threshold functions that did not deteriorate over time was the psychophysical data. It was a pleasant surprise that it "worked." As the amount of sensory response remaining in the memory traces decayed with simulated time, the difference-thresholds generally increased. Figure 16 shows that, when averaged across levels, the difference-thresholds increased like decay functions. (The decay functions end at the 64 memory time for clarity and to keep the functions in the same 


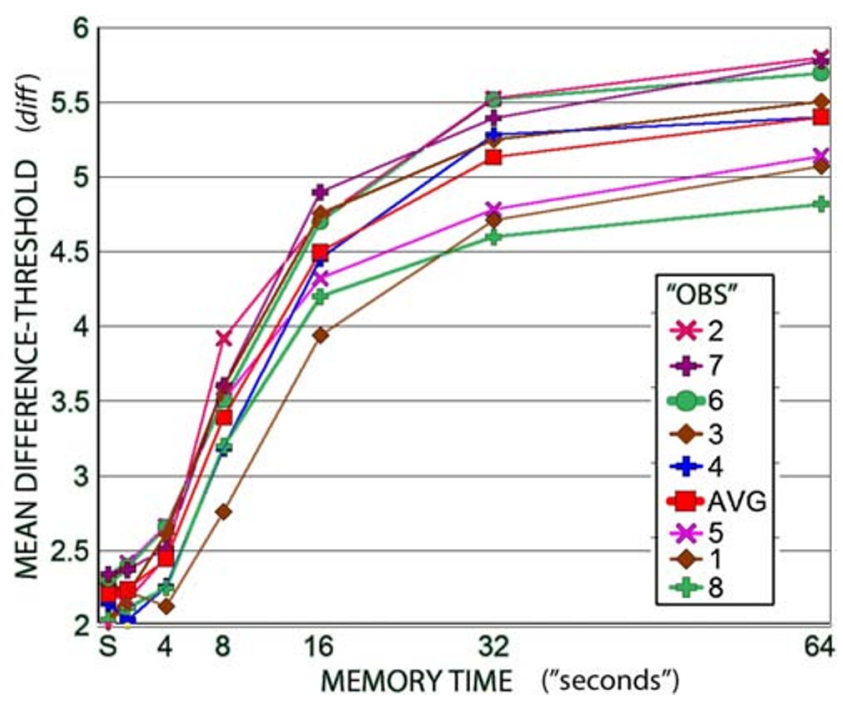

Fig. 16 The model's difference-thresholds are averaged across the 16 stimulus levels to show their decay as a function of simulated memory time from simultaneous matches (S) to matches made after 64 "s". The results are for eight independent "observers" and their mean

range with respect to half-life for comparison with the experimental decay functions.) As expected the average decay function doubled in mean difference-threshold at about the designed half-life time of $16 \mathrm{~s}$ - though there were notable "individual" differences.

Despite this decay the individual "observer" memory difference-threshold functions tended to resemble the non-decayed "sensory" function at memory times up to 32 "s" - twice the half-life (see Fig. 17). Figure 17 RIGHT shows that the average observer function loses that similarity at the 32-s delay and beyond because increasing "individual" differences averaged out their distinctive shapes. This is also evident in the experimental average observer functions in Experiments 1-5. Figure 17 left shows that maxima and minima shifted as a function of stimulus level for "observer" number 6. Similar shifts occurred for observers in Experiments 1-5. For example, HAN's results in Fig. 13 show notable peak shifts at orientations between $25^{\circ}$ and $65^{\circ}$ as memory time increased.

Table 2 shows the similarity of the "memory" differencethresholds to the non-decayed "sensory" functions for the eight "observers" and their average. This similarity began to decrease markedly as "memory" extended beyond twice the half-life of $16 \mathrm{~s}$. Note how the results for observer number 6 (Fig. 17, left) changed as memory time extended beyond twice the half-life time. This is consistent with Perez-Carpinell et al.'s (1998) results (Fig. 5) as memory time extended beyond $15 \mathrm{~s}$. Whether such changes at longer memory times arise just from decay as suggested by the model or represent transition to another process remains to be determined.

The model demonstrates that reducing the distinctiveness of a stored pattern by adding randomness does not necessarily prevent a recognition-by-correlation process from revealing that pattern through the differencethresholds obtained from the standard deviations of repeated matching attempts. Extending the model to when

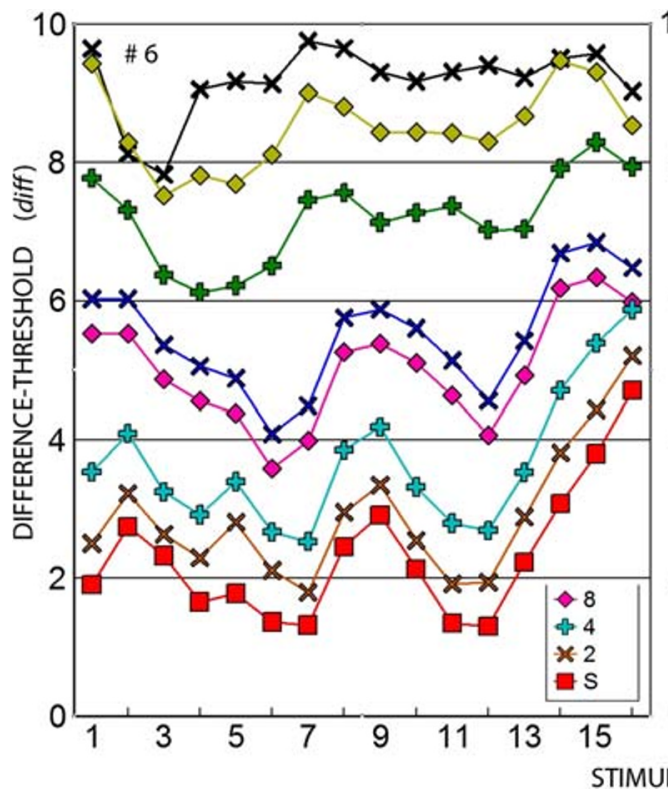

Fig. 17 Simulated difference-thresholds in relative diff values as a function of 16 stimulus levels. Left: The results for "observer" number 6 based on eight matches at each condition. Right: The results averaged for eight "observers." The bottom functions (S) were obtained by

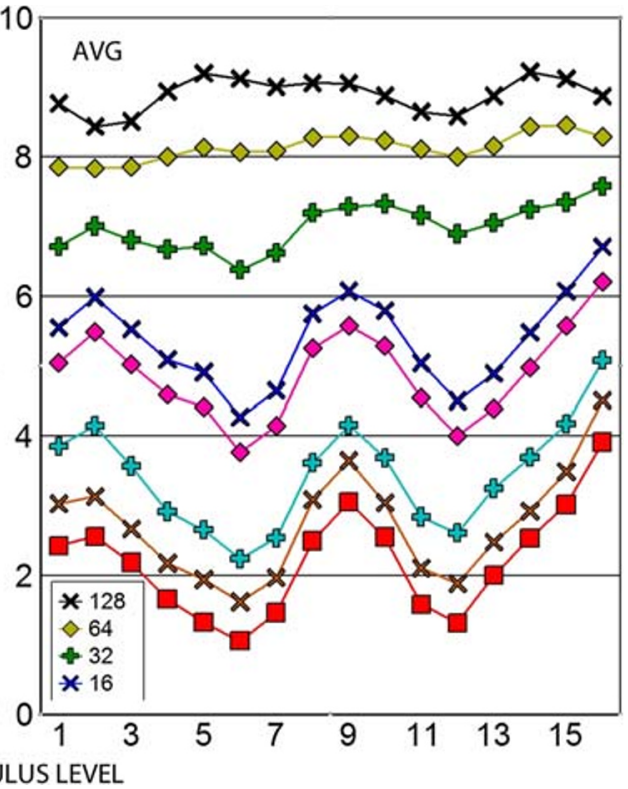

matching a non-decayed "sensory" response to various comparison stimuli. The seven upper functions were obtained similarly by matching "memory" traces at the $2,4,8,16,32,64$, and 128 -s memory times. The memory functions are successively offset by 0.5 diff 
Table 2 Correlation of the memory difference-threshold functions with their non-decayed sensory function based on eight matches per memory time $\times$ level condition by each "observer" at simulated memory times from $2 \mathrm{~s}$ to $128 \mathrm{~s}$, with a half-life proportionally set at time 16 (with 16 levels per function, the 14 d.f. have a $95 \%$ confidence of similarity $=$ $+0.50)$

\begin{tabular}{|c|c|c|c|c|c|c|c|c|c|}
\hline \multirow{2}{*}{$\begin{array}{l}\text { Memory } \\
\text { Time }\end{array}$} & \multicolumn{8}{|c|}{ Observers } & \multirow{2}{*}{$\begin{array}{l}\text { Avg } \\
\text { Obs }\end{array}$} \\
\hline & 1 & 2 & 3 & 4 & 5 & 6 & 7 & 8 & \\
\hline 2 & 0.98 & 0.96 & 0.91 & 0.90 & 0.90 & 0.86 & 0.91 & 0.97 & \\
\hline 4 & 0.93 & 0.90 & 0.83 & 0.73 & 0.88 & 0.83 & 0.79 & 0.73 & 0.9 \\
\hline 8 & 0.81 & 0.77 & 0.65 & 0.61 & 0.83 & 0.65 & 0.80 & 0.55 & \\
\hline 16 & 0.29 & 0.75 & 0.63 & 0.55 & 0.80 & 0.61 & 0.77 & 0.49 & \\
\hline 32 & 0.29 & 0.81 & 0.54 & 0.47 & 0.77 & 0.56 & 0.73 & 0.46 & \\
\hline 64 & 0.22 & 0.74 & 0.52 & 0.44 & 0.74 & 0.62 & 0.66 & 0.46 & 0.8 \\
\hline 128 & 0.26 & 0.69 & 0.49 & 0.46 & 0.68 & 0.60 & 0.58 & 0.26 & 0.7 \\
\hline Mean & 0.55 & 0.80 & 0.65 & 0.59 & 0.80 & 0.68 & 0.75 & 0.56 & 0.8 \\
\hline
\end{tabular}

decay leaves only $10 \%$ of the STIMULUS TO REMEMBER showed that 64 matches still produced a significant mean similarity over 60 trials of +0.54 (at 14 d.f. for the 16 levels modeled, $95 \%$ confidence $=+$ $0.50)$. Standard deviations of multiple matches are a second-moment analog of how signal averaging extracts signals from noise. While this does not prove that the consistency of memory difference-thresholds is based on sensory-like responses from memory, it does demonstrate the possibility.

\section{Conclusion}

When monochromatic hues and the orientation of lines and gratings were repeatedly matched directly, successively, or after various delays up to $24 \mathrm{~s}$, the means of these matches did not change significantly as a function of time. This suggests that the variability of the repeated measurements was due to noise - not to shifts in memory. The variability of the matches increased with time in a manner resembling exponential decay. The half-life of the decay functions differed for hue, line orientation, and grating orientation. As discussed previously, these differences are consistent with a greater stability of memories that involve more information (Becker et al., 2013).

Since variability of stimulus matches is proportional to their just-noticeable difference, difference-thresholds were obtained as a function of wavelength and orientation. Despite the overall increase in variability indicated by the decay functions, the memory difference-threshold functions did not fade as memory time increased. Rather, they continued to resemble the sensory functions. This resemblance was confirmed by significant correlations of the memory to the sensory functions. This resemblance was not attributable to averaging results across observers, a retinal effect, or the method of measuring difference-thresholds. This resemblance has also been found to a limited extent in a few other psychophysical experiments that have measured memory differencethresholds for color (Bloj et al., 2016; Nilsson \& Nelson, 1981; Perez-Carpinell et al., 1998), orientation (Balikou et al., 2015; Jastrow, 1892; Pratte et al., 2017; Vogels \& Orban, 1986), and spatial frequency contrast (Lee \& Harris, 1996).

How could difference-threshold functions retain their characteristic shape while what was stored in memory decayed as indicated by the increasing variability of the matches? The lack of a shift in the matches made from memory indicates the variability was random. It is well established that averaging is an effective means of extracting a signal from noise (e.g., Dawson, 1954; Regan, 2009). Perhaps the retention arose from the averaging inherent in calculating second-moment standard deviations of repeated matches. To test this possibility, a neural network model of the memory-matching procedure was created. This model demonstrated that differencethresholds calculated from memory matches resembled difference-thresholds calculated from the original signal even when only $17 \%$ of the stimulus remained in memory. Since a system's response to a series of levels can be derived by integrating its difference-thresholds as a function of those levels, it is reasonable to conclude that memory difference-threshold functions reflect the system's memory response to those levels. This suggests that similarity of sensory-sensory to sensory-memory comparisons involves comparisons of similar system responses.

Even as memory difference-thresholds decay with time, they provide an "inside scoop" on the conscious processing that makes recognition-matching possible (Baumann, Endestad, \& Magnussen, 2008; Fallon, Zokaei, \& Husain, 2016; Harrison \& Tong, 2009; Jacobs \& Silvanto, 2015). This corroborates correlational evidence from cortical recordings of a comparison process (Cichy et al., 2019; Yin et al., 2012) based on memory activity that resembles sensory responses (e.g., functions found by Balikou et al., 2015; Ester, Anderson, Serences, \& Awh, 2013; Honkanen et al., 2015; Peterson et al., 2014; Sneve et al., 2012; Sreenivasan, Curtis, \& D'Esposito, 2014; Thakral \& Slotnik, 2015). It provides a simple means of maintaining perceptual stability across saccades (Cronin \& Irwin, 2018), and explains how recall can be so rapid (e.g., Clark \& Gronlund, 1996; DiCarlo, Zoccolan, \& Rust, 2012) or so efficient (Gayet et al., 2016) even when a recognized stimulus only partially resembles the remaining memory of the original (Song \& Jiang, 2005). These diverse lines of psychophysical, neural model, and 
cortical measurements justify concluding that what came out of memory was similar to the sensory response producing that memory.

Acknowledgements In memory of the late Thomas M. Nelson, Professor Emeritus, University of Alberta - mentor, colleague, friend: This research was possible thanks to 23 University of Prince Edward Island undergraduate students whose careful and patient observations provided the data. It was supported by grants from the National Science and Engineering Research Council of Canada, and the Maritime Provinces Higher Education Commission. Some of the apparatus was graciously loaned by the University of Alberta's Psychology Department. The data for all experiments and the memory match model are available in QuattroPro spreadsheets from open Science Frame (https://doi.org/10.17605/OSF. IO/9XE4S) and the author (https://www.nilsson@upei.ca). None of the experiments were preregistered.

\section{Appendix}

\section{Memory Decay Model}

The model runs on Corel's QuattroPro X8 and earlier versions. It is available by e-mail as a .qpw file from the author and from the Open Science Framework. Figure 18 illustrates the model. To fit into a publishable figure size and remain legible, the illustration differs from the actual model by: (1) not showing columns (AD to $\mathrm{AQ}$ ) which include correlations for the 16, 32, 64, and 128 memory times; (2) cutting off columns from BC to $\mathrm{DK}$, which contain the correlation calculations for comparison stimuli differences of +6 to -8 from the stimulus response; and (3) not showing rows below 94, which contain the stimuli for the various levels, a summary of the results, calculations of the similarity of the memory difference thresholds to the "sensory" difference-thresholds, and graphs of the results.

Seven sections of the spreadsheet's operations are illustrated:

1. Upper left - This section contains in cells B7-B19 a 13-part set of randomly generated numbers which represents the STIMULUS TO REMEMBER. This section also contains in modifiable yellow cells: random number seeds for memory noise (G4) and sensor sensitivity (G6), the number of matches (G8), a memory gain factor (F11), a memory noise gain factor (G13), a sensory transducer noise factor (G16), a comparison stimulus difference factor (H18). Where these factors are used in the spreadsheet is noted by the arrows to their right. The 13-part set of green cells at $\mathrm{O} 7-\mathrm{O} 19$ contains randomly generated noise values which get added to the respective stimulus parts in the memory traces by increasing proportion as memory time increases - leaving only $50 \%$ of the stimulus response in the trace at memory time 16.
2. Mid left - The block of blue numbers at B26-N42 holds the COMPARISON STIMULI to be compared with the sensory and memory traces of the STIMULUS TO REMEMBER. Arranged in rows, the values of the comparison stimuli represent from one to eight difference steps that are added or subtracted from the response of the stimulus being recalled. The center row (blue field) has transducer modified response values of the stimulus being remembered. Below them in B47 to N54 are calculated the stimulus differences used for the comparison stimuli.

3. Bottom left - The violet boxes contain the macro programs that conduct correlating the comparison stimuli with the sensory and memory traces to obtain the number of matches specified in G8. The yellow cells at B90-Q90 contain modifiable random number seeds to vary the transducer sensitivity to the various stimulus levels. Larger values tend to increase the size of the COMPARISON STIMULI difference steps (diff), thereby making matches to smaller difference steps more likely to be the best match.

4. Upper center - This sets up the 13-part sensory responses (R7-R19) and memory traces at times 2-8 (U7-U19, X7-X19, AA7-AA19) for entry into the correlation matrix. Traces for memory times $16-128$ in columns AD to AM are not shown. The memory traces accumulate more of the 13part noise values as memory time increases

5. Mid center - The red numbers at R26-R42 are the correlations of each comparison stimulus with the stored sensory response. The correlations of each comparison stimulus with the two, four, and eight memory time traces are in $\mathrm{U} 26-\mathrm{U} 42, \ldots, \mathrm{AA} 42$. Correlations for memory times $16-128$ in columns AD-AM are not shown. This region selects for a match the diff value of the comparison stimulus which correlates highest with the trace at each memory time.

In the orange field, columns at S46-S50, V46-V50, ..., AB50 accumulate the diff values of the best comparison stimuli and calculates their difference-threshold in terms of their standard deviation using the following formula:

$\mathrm{SD}=\left(\left(\sum \mathrm{x}^{2}\right)-\overline{\mathrm{x}} 2\right)^{0.5}$

6. Bottom center - MACRO B repeats the differencethreshold measurements for 16 stimulus levels.

7. Right side - Columns AV-DN (far right, not shown) calculate the correlations as the average product of the Zvalues of the comparison stimuli and memory traces. 


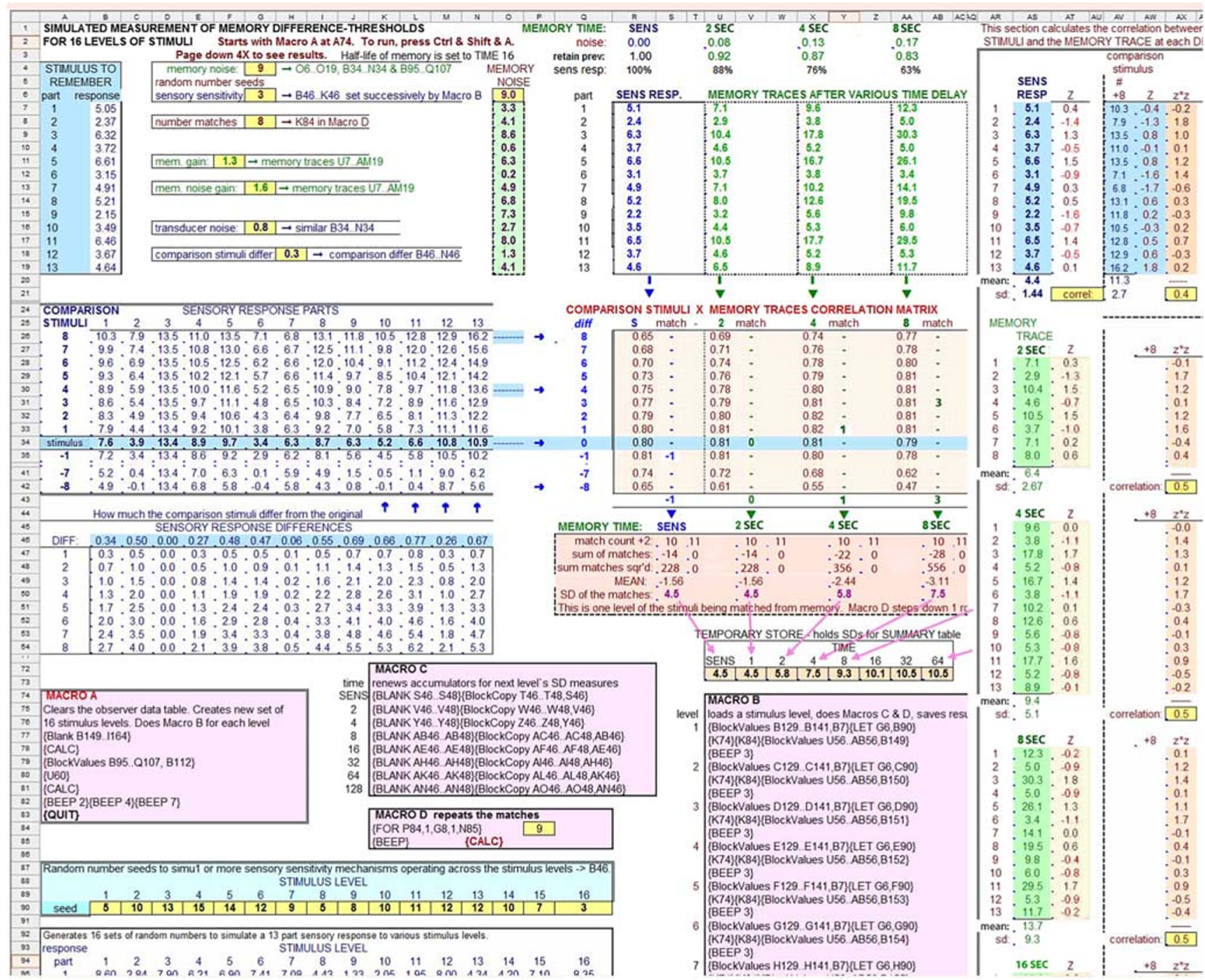

Fig. 18 Illustration of the QuattroPro spreadsheet that generates memory difference-thresholds 


\section{References}

Allen, E. C., Beilock, S. L., \& Shevell, S. K. (2011). Working memory is related to perceptual processing: A case from color perception. Journal of Experimental Psychology: Learning, Memory \& Cognition, 37, 1014-1021.

Amtul, Z., \& Rahman, A. (2016). Neural plasticity and memory: Is memory encoded in hydrogen bonding patterns? The Neuroscientist, 22, 9-18.

Andrews, D. P. (1967). Perception of contour orientation in the central fovea. Part I: Short lines. Vision Research, 7, 975-997.

Austin, J., \& Lees, K. (2000). A search engine based on neural correlation matrix memories. Neurocomputing, 35, 55-72.

Baddeley, A. D., \& Scott, D. (1971). Short term forgetting in the absence of proactive interference. Quarterly Journal of Experimental Psychology, 23, 275-283.

Bae, G. Y., \& Luck, S. J. (2017). Interactions between visual working memory representations. Attention, Perception \& Psychophysics, 79, 2376-2395.

Bae, G. Y., Olkkonen, M., Allred, S. R. \& Flombaum, J. I. (2015). Why some colors appear more memorable than others: A model combining categories and particulars in color working memory. Journal of Experimental Psychology: General, 144, 744-763.

Balikou, P., Gourtzelidis, P., Mantas, A., Moutoussis, K. Evdokimidis, I., \& Smyrnis, N. (2015). Independent sources of anistropy in visual orientation representation: A visual and a cognitive effect. Experimental Brain Research, 233, 3097-3108.

Baumann, O., Endestad, T., \& Magnussen, S. (2008). Delayed discrimination of spatial frequency for gratings of different orientation: behavioral and fMRI evidence for low-level perceptual memory stores in early visual cortex. Experimental Brain Research 188, 363-369.

Becker, M. W., Miller, J. R., \& Liu, T. (2013). A severe capacity limit in the consolidation of orientation information into visual short-term memory. Attention, Perception \& Psychophysics, 75, 415-425.

Bentley, I. M. (1899). The memory image and its qualitative fidelity. The American Journal of Psychology, 11, 1-48.

Blackwell, H. R. (1972). Luminance difference thresholds. In D. Jameson \& L. M. Hurvich (Eds.). Handbook of Sensory Physiology, Vol. VII/ 4, Visual Psychophysics (pp. 78-101). New York: Springer-Verlag.

Blakemore, C., \& Campbell, F. W. (1969). On the existence of neurons in the human visual system selectively sensitive to the orientation and size of retinal images. Journal of Physiology, 203, 237-260.

Bloj, M., Weiss, D., \& Gegenfurtner, K. R. (2016). Bias effects of shortand long-term memory for unique objects. Journal of the Optical Society of America - A, 33, 492-500.

Bornstein, M. H. (1973). Color vision and color naming. Psychological Bulletin, 80, 257-285.

Bouman, M. A., \& Walraven, P. L. (1972). Color discrimination data. In D. Jameson \& L. M. Hurvich (Eds.). Handbook of Sensory Physiology, Vol. VII/4, Visual Psychophysics (pp. 484-516). New York: Springer-Verlag

Bradley A., \& Skottun, B. C. (1984). The effects of large orientation and spatial frequency differences on spatial discrimination. Vision Research, 24, 1889-1896.

Brown, J. (1958). Some tests of the decay theory of immediate memory. Quarterly Journal of Experimental Psychology, 10, 12-21.

Brown, P. K., \& Wald, G. (1964). Visual pigments in single rods and cones of the human retina. Science, 144, 45-52.

Burbeck, C. A., \& Regan D. (1983). Independence of size and orientation in spatial discriminations. Journal of the Optical Society of America, 73, 1691-1694.

Campbell, F. W., \& Kulikowski, J. J. (1966). Orientation selectivity of the human visual system. Journal of Physiology, 187, 437-445.
Campbell, F. W., Kulikowski, J. J., \& Levinson, J. (1966). The effect of orientation on the visual resolution of gratings. Journal of Physiology, 187, 427-436.

Cattaneo, Z., Vecchi, T., Pascual-Leone, A., \& Silvanto, J. (2009). Contrasting early visual cortical activation states causally involved in visual imagery and short-term memory. European Journal of Neuroscience, 30, 1393-1400.

Caziot, B., Valsecchi, M., Gengenfurtner, K. R., \& Backus, B. T. (2015). Fast perception of binocular disparity. Journal of Experimental Psychology: Human Perception and Performance, 41 (4), 909-916.

Chapanis, A. (1951). Studies of manual rotary positioning movements: II The accuracy of estimating the position of an indicator knob. The Journal of Psychology, 31, 65-71.

Cichy, R. M., Kriegeskorte, N., Jozwik, K. M., van den Bosch, J. J. F., \& Charest, I. (2019). The spatiotemporal dynamics underlying perceived similarity for real-world objects. Neuroimage, 194, 12-24.

Clark, S. E., \& Gronlund, S. D. (1996). Global matching models of recognition memory: How the models match the data. Psychonomic Bulletin \& Review, 3, 37-60.

Collins, M. (1932). Some observations of immediate colour memory. British Journal of Psychology, 22, 344-352.

Cook, E. W., \& Miller, G. A. (1992). Digital filtering: Background and tutorial for psychophysiologists. Psychophysiological Research, 29, 350-367.

Cornelissen, F. W., \& Greenlee, M. W. (2000). Visual memory for random block patterns defined by luminance and color contrast. Vision Research, 40, 287-299.

Cronin, D. A., \& Irwin, D. E. (2018). Visual working memory supports perceptual stability across saccadic eye movements. Journal of Experimental Psychology, 44, 1739-1759.

Dara, R., Satyanarayana, Ch., \& Govardhan, A. (2014). A novel approach for image recognition to enhance the quality of decision making by applying degree of correlation using artificial neural networks. International Journal of Image, Graphics and Signal Processing, $11,25-35$.

Dawson, G. D. (1954). A summation technique for the detection of signals in noise. EEG \& Clinical Neurophysiology, 6, 65-84.

De Valois, K. K., De Valois, R. L., \& Yund, W. W. (1979). Responses of striate cortex cells to grating and checkerboard patterns. Journal of Physiology, 291, 483-505.

DiCarlo, J. J., Zoccolan, D., \& Rust, N. C., (2012). How does the brain solve visual object recognition? Neuron, 73, 415-434.

Eichengren, J. M. (1976). Unique hue loci: Induced shifts with complimentary surrounds. Vision Research, 16, 199-204.

Ermentrout, G. B., Galan, R. F., Urban N. N., (2008). Reliability, synchrony and noise. Trends in Neuroscience, 31, 428-434.

Ester, E. F., Anderson, D. E., Serences, J. T., \& Awh, E., (2013). A neural measure of precision in visual working memory. Journal of Cognitive \& Neural Science, 25, 754-761.

Etzkorn, B. (2012). Data normalization and standardization. www. Benetzetzkorn.com/wp-content/uploads/2011/11/DataNormalization-and-Standardization.pdf

Fahle, M. F., \& Harris, J. P. (1992). Visual memory for vernier offsets. Vision Research, 32, 1033-1042.

Fallon, S. J., Zokaei, N., \& Husain, M. (2016). Causes and consequences of limitations in visual working memory. Annals of the New York Academy of Sciences, 1369, 40-54.

Foster, D. H., \& Westland, S. (1995). Orientation contrast vs orientation in line-target detection. Vision Research, 35, 733-738.

Foster, K. H., Gaska, J. P., Nagles, M., \& Pollen, D. H. (1985). Spatial and temporal frequency selectivity of neurons in areas V1 and V2 of the macaque monkey. Journal of Physiology, 365, 331-363.

Furmanski, C. S., \& Engel, S. A. (2000). An oblique effect in human primary visual cortex. Nature Neuroscience, 3, 535-536.

Fuster, J. M. (1997). Network memory. Trends in Neurosciences, 20, 451458. 
Gayet, S., van Maanen, L., Heilbron, M., Paffen, C. L. E., \& van der Stigchel, S. (2016). Visual input that matches the content of visual working memory requires less (not faster) evidence sampling to reach conscious access. Journal of Vision, 16(11) 26, 1-20.

Graham, C. H. (1965). Color: Data and theories. In C. H. Graham (Ed.). Vision and Visual Perception (pp. 414-451). New York: John Wiley $\&$ Sons.

Graham, N. V. S. (1989). Neurophysiology and psychophysics. In Visual Pattern Analyzers, Oxford University Press USA - OSO ProQuest Ebook Central (pp. 3-34). https://ebookcentral.proquest.com/lib/ upei/detail. action?docID=3051922.

Guo, Z. V., Inagaki, H. K., Daie, K., Druckmann, S., Gerfen, C. R., \& Svoboda, K. (2017). Maintenance of persistent activity in a frontal corticothalamical loop. Nature, 545, 181-186.

Harrison, S. A., \& Tong, F. (2009). Decoding reveals the contents of visual working memory in early visual areas. Nature, 458, 510-519.

Heeley, D. W., \& Timney, B. (1988). Meridional anisotropies of orientation discrimination for sine wave gratings. Vision Research, 88, $337-$ 344.

Hole, G. J. (1996). Decay and interference effects in visuospatial memory. Perception, 25, 53-64.

Honkanen, R., Rouhinen, S., Wang, S. H., Palva, J. M., \& Palva, S. (2015). Gamma oscillations underlie the maintenance of featurespecific information and the contents of visual working memory. Cerebral Cortex, 25, 3788-3801.

Hubel, D. H., \& Weisel, T. N. (1959). Receptive fields of single neurones in the cat's striate cortex. Journal of Physiology, 148, 574-591.

Jacobs, C., \& Silvanto, J. (2015). How is working memory content consciously experienced? The 'conscious copy' model of WM introspection. Neuroscience and Biobehavioral Reviews, 55, 510-519.

Jastrow, J. (1892). On the judgement of angles and positions of lines. American Journal of Psychology, 11, 214-217.

Kaiser, P. K., \& Boynton, R. M. (1996). Chromatic Discrimination. In P. K. Kaiser \& R. M. Boynton (Eds.). Human color vision 2nd (pp. 312-356). Washington, D.C.: Optical Society of America.

Killebrew, K. W., Gurariy, G., Peacock, C. E., Berryhill, M. E., \& Caplovitz, G. P. (2018). Electrophysiological correlates of encoding processes in a full-report visual working memory paradigm. Cognitive, Affective \& Behavioral Neuroscience, 18, 353-365.

Laming, D., \& Laming, J. (1952). F. Hegelmaier: on memory for the length of a line. Psychological Research, 54, 233-239.

Laming, D. \& Scheiwiller, P. (1985). Retention in perceptual memory: A review of models and data. Perception \& Psychophysics, 37 (3), 189-197.

Lee, B., \& Harris, J. (1996). Contrast transfer characteristics of visual short-term memory. Vision Research, 36, 2159-2166.

Long, G. M., \& O'Saben, C. L. (1989). The changing face of visual persistence. American Journal of Psychology, 102, 197-210.

Macho, S. (2002). Cognitive modeling with spreadsheets. Behavior Research Methods, Instruments, \& Computers, 34, 19-34.

Maffei, L., \& Fiorentini, M. (1973). The visual cortex as a spatial frequency analyser. Vision Research, 13, 1255-1267.

Magnussen, S. (2009). Implicit visual working memory. Scandinavian Journal of Psychology, 50, 535-542.

Magnussen, S., Idas, E., \& Myhre, S. H. (1998). Representation of orientation and spatial frequency in perception and memory: A choice reaction-time analysis. Journal of Experimental Psychology: Human Perception \& Performance, 24, 707-718.

Magnussen, S., Landro, N. I., \& Johnsen, T. (1985). Visual half-field symmetry in orientation perception. Perception, 14, 265-273.

Mansfield, R. (1974). Neural basis for orientation perception in primate vision. Science, 186, 133-135.

Marks, W. B., Dobelle, W. H., \& MacNichol, E. F. (1964). Visual pigments of single primate cones. Science, 143, 1181-1183.
Melton, A. W. (1963). Implications of short-term memory for a general theory of memory. Journal of Verbal Learning and Verbal Behavior, $2,1-21$.

Mercer, T. (2014). The loss of short-term visual representations over time: Decay of temporal distinctiveness? Journal of Experimental Psychology: Human Perception and Performance, 40, 2281-2288.

Metcalfe, J. (1991). Recognition failure and the composite memory trace in CHARM. Psychological Review, 98, 529-553.

Mewhort, D. J. K., \& Johns, E. E. (2005). Sharpening the echo: An iterative-resonance model for short-term recognition memory. Memory, 13, 300-307.

Mikhailova, E. S., Gerasimenko, N. Y., Slavutskaya, V., Krylova, M. A., \& Izyurov, I. V. (2017). Temporal and topographic characteristics of evoked potentials in the conflict of two consecutive visual stimuli in a working memory task. Human Physiology, 43, 248-258.

Nemes, V. A., Parry, N. R. A., \& McKeefry, D. J. (2010). A behavioural investigation of human visual short term memory for colour. Ophthalmic \& Physiological Optics, 30, 594-601.

Nilsson, T. H. (1971). Device producing two beams of different wavelength from single monochromator. Applied Optics, 10, 2561.

Nilsson, T. H., (1979). Visual temporal discrimination of brief pulse intervals. Vision Research, 19, 41-48.

Nilsson, T. H., \& Nelson, T. M. (1981). Delayed monochromatic hue matches indicate characteristics of visual memory. Journal of Experimental Psychology: Human Perception and Performance, 7, 141-150.

Nilsson T. H., Richmond C. F., \& Nelson T. M. (1975). Flicker adaptation shows evidence of visual channels selectively sensitive to temporal frequency. Vision Research, 15, 621-624.

Ogilvie, J. C., \& Taylor, M. M. (1958). Effect of orientation on the visibility of fine lines. Journal of the Optical Society of America, 48, 628-629.

Orban, G. A., Vandenbussche, E., \& Vogels, R. (1984). Human orientation discrimination tested with long stimuli. Vision Research, 24, 121-128.

Parks, T. E., Kroll, N. E. A., Salzberg, P. M., \& Parkinson, S. R. (1972). Persistence of visual memory as indicated by decision time in a matching task. Journal of Experimental Psychology, 92, 437-438.

Pasternak, T., \& Greenlee, M. W. (2005). Working memory in primate sensory systems. Nature Reviews: Neuroscience, 6, 97-106.

Perez-Carpinell, J., Baldovi, R., de Fez, M. D., \& Castro, J. (1998). Color memory matching: Time effect and other factors. Color Research and Application, 23, 234-247.

Pergola, G., Ranft, A., Mathias, K., \& Suchan, B. (2013). The role of thalamic nuclei in recognition memory accompanied by recall during encoding and retrieval: An fMRI study. Neuroimage, 74, 195 208.

Pertzov, Y., Manohar, S., \& Husain, M. (2016). Rapid forgetting results from competition over time between items in visual working memory. Journal of Experimental Psychology: Learning, Memory, \& Cognition, 43, 528-536.

Peterson, D. J., Gurariy, G., Dimotsantos, G. G., Arciniega, H., Berryhill, M. E., \& Caplovitz, G. P. (2014). The steady-state visual evoked potential reveals neural correlates of the items encoded into visual working memory. Neuropsychologia, 63, 145-153.

Petrusic, W. M., Harrison, D. H., \& Baranski, J. V. (2004). Long-term memory for elementary visual percepts: memory psychophysics of context and acquisition effects. Perception \& Psychophysics, 66, 430-445.

Pokorny, J., \& Smith, V. C. (2004). Chromatic Discrimination. In L. M. Chalupa \& J. S. Werner (Eds.). The Visual Neurosciences (pp. 908923). Cambridge, MA: MIT Press.

Postle, B. R. (2015). The cognitive neuroscience of visual short-term memory. Current Opinion in the Behavioral Sciences, 1, 40-46.

Pratte, M. S., Park, Y. E., Rademaker, R. L. \& Tong F. (2017). Accounting for stimulus specific variation in precision reveals a discrete capacity 
limit in vision. Journal of Experimental Psychology: Human Perception \& Performance, 43, 6-17.

Pribram, K. H., Nuwer, M., \& Baron, R. J. (1974). The holographic hypothesis of memory structure in brain function and perception. In D. H. Krantz, R. C. Atkinson, D. Luce \& P. Suppes (Eds.). Contemporary Developments in Mathematical Psychology, 2 (pp. 416-457). San Francisco: W. H. Freeman.

Rademaker, R. L., Park, Y. E., Sack, A. T., \& Tong, F. (2018). Evidence of gradual loss of precision for simple features and complex objects in visual working memory. Journal of Experimental Psychology: Human Perception \& Performance, 44, 925-940.

Rademaker, R. L., van den Ven, V. G., Tong, F., \& Sack, A. T. (2017). The impact of early visual cortex transcranial magnetic stimulation on visual working memory precision and guess rate. PLoS ONE 12(4): e0175230. https://doi.org/10.1371/journal.pone0175230

Regan, D. (2009). Some early uses of evoked brain responses in investigation of human visual function. Vision Research, 49, 882-897.

Ricker, T. J., Spiegel, L. R., \& Cowan, N. (2014). Time based loss in visual short-term memory is from trace decay, not temporal distinctiveness. Journal of Experimental Psychology: Learning, Memory, \& Cognition, 40, 1510-1523.

Riggs L. A., \& Wooten, B. R. (1972). Electrical measures and psychophysical data on human vision. In D. Jameson \& L. M. Hurvich (Eds.). Handbook of Sensory Physiology, Vol. VII/4, Visual Psychophysics (pp. 690-731). New York: Springer-Verlag.

Sakai, K. (2003). Short-term visual memory for contour curvature in delayed discrimination task. Japanese Psychological Research, 45, 122-128.

Sakitt, B. (1975). Locus of short-term visual storage. Science, 190, 13181319.

Schneegans, S., \& Bays, P. M. (2018). Drift in neural population activity causes working memory to deteriorate. Journal of Neuroscience, 38 , 4859-4869.

Schurgin, M. W. (2018). Visual memory, the long and the short of it: A review of visual working memory and long-term memory. Attention, Perception \& Psychophysics, 80, 1035-1056.

Seliger, H. H. (2002). Measurement of the memory of color. Color Research and Application, 27, 233-242.

Serences, J. T. (2016). Neural mechanisms of information storage in visual short-term memory decay. Vision Research, 128, 53-67.

Shin, H., Zou, Q., \& Ma, W. J. (2017). The effects of delay duration on visual working memory for orientation. Journal of Vision, 17(14), 10, 1-24.

Skottun, B. C. (2004). On the use of discrimination to assess memory. Perception \& Psychophysics, 66, 1202-1205.

Sneve, M. H., Alnaes, D., Endestad, T., Greenlee, M. W., \& Magnussen, S. (2012). Visual short-term memory: Activity supporting encoding and maintenance in retinotopic visual cortex. NeuroImage, 63, 166178.

Sneve, M. H., Sreenivasan, K. K., Alnaes, D., Endestad, T., \& Magnussen, S. (2015). Short-term retention of visual information: Evidence in support of feature-based attention as an underlying mechanism. Neuropsychologia, 66, 1-9.
Song, J-H., \& Jiang, Y. (2005). Connecting the past with the present: How do humans match an incoming visual display with visual memory? Journal of Vision, 5, 322-330.

Souza, A. S., \& Oberauer, K. (2015). Time-based forgetting in visual working memory reflects temporal distinctiveness not decay. Psychonomic Bulletin \& Review, 22, 156-162.

Souza, A. S., \& Oberauer, K. (2017). The contributions of visual and central attention to visual working memory. Attention, Perception \& Psychophysics, 78, 1897-1916.

Sreenivasan, K. K., Curtis, C. E., \& D'Esposito, M. (2014). Revisiting the role of persistent neural activity during working memory. Trends in Cognitive Science, 18, 82-89.

Stiles, W. S. (1959). Color vision: The approach through incrementthreshold sensitivity. Proceedings of the National Academy of Sciences, USA, 45, 100-114.

Thakral, P. P., \& Slotnik, S. D. (2015). The sensory timecourses associated with conscious visual item memory and source memory. Behavioural Brain Research, 290, 143-151.

Tonegawa, S., Liu, X., Ramirez, S., \& Redondo, R, (2015). Memory engram cells have come of age. Neuron, 87, 907-931.

Uchikawa, K., \& Ikeda, M. (1981). Temporal deterioration of wavelength discrimination with successive comparison method. Vision Research, 21, 591-595.

van Vugt, M. K., Sekuler, R., Wilson, H. R., \& Kahana, M. J. (2013). An electrophysiological signature of summed similarity to visual working memory. Journal of Experimental Psychology: General, 142, 412-425.

Vogels, R., \& Orban, G. A. (1986). Decision processes in visual discrimination of line orientation. Journal of Experimental Psychology: Human Perception and Performance, 12, 115-132.

Westheimer, G., \& Beard, B. F. (1998). Orientation dependency for foveal line stimuli: Detection and intensity discrimination, resolution, orientation discrimination and vernier acuity. Vision Research, 38, 1097-1103.

Wickelgren, W. A. (1974). Strength / resistance theory of the dynamics of memory storage. In D. H. Krantz, R. C. Atkinson, R. D. Luce, \& P. Suppes (Eds.). Contemporary Developments in Mathematical Psychology - Volume I: Learning, Memory, and Thinking, (pp. 209-242). San Francisco: W. H. Freeman.

Woodworth, R. S., \& Schlosberg, H. (1954). The method of average error. In Experimental Psychology (pp. 199-200). New York: Holt, Rinehart \& Winston.

Wright, W. D., \& Pitt, F. H. G. (1934). Hue-discrimination in normal colour-vision. Proceedings of the Physical Society, 46, 459-468.

Yin, J., Gao, Z., Jin, X., Ding, X, Liang, J., \& Shen, M. (2012). The neural mechanisms of percept-memory comparison in visual working memory. Biological Psychology, 90, 71-79.

Zhang, Q. (2008). A computational account of dreaming: Learning and memory consolidation. Cognitive Systems Research, 10, 91-101.

Zhang, W., \& Luck, S. J. (2011). The number and quality of representations in working memory. Psychological Science, 22, 1434-1441.

Publisher's note Springer Nature remains neutral with regard to jurisdictional claims in published maps and institutional affiliations. 